\title{
Different Munc18 proteins mediate baseline and stimulated airway mucin secretion
}

\author{
Ana M. Jaramillo, ${ }^{1,2}$ Lucia Piccotti, ${ }^{1}$ Walter V. Velasco, ${ }^{1}$ Anna Sofia Huerta Delgado, ${ }^{3}$ \\ Zoulikha Azzegagh, ${ }^{1}$ Felicity Chung, ${ }^{4}$ Usman Nazeer, Junaid Farooq, ${ }^{1}$ Josh Brenner, \\ Jan Parker-Thornburg, ${ }^{5}$ Brenton L. Scott, ${ }^{1}$ Christopher M. Evans, ${ }^{6}$ Roberto Adachi, ${ }^{1}$ \\ Alan R. Burns, ${ }^{7}$ Silvia M. Kreda, ${ }^{4}$ Michael J. Tuvim, ${ }^{1}$ and Burton F. Dickey ${ }^{1}$
}

'Department of Pulmonary Medicine, University of Texas MD Anderson Cancer Center, Houston, Texas, USA. ${ }^{2}$ Institute of Bioscience and Technology, Texas A\&M University Health Science Center, Houston, Texas, USA. ${ }^{3}$ Tecnologico de Monterrey, Escuela de Medicina y Ciencias de la Salud, Monterrey, Mexico. ${ }^{4}$ Marsico Lung Institute/Cystic Fibrosis Center, University of North Carolina at Chapel Hill, Chapel Hill, North Carolina, USA. ${ }^{5}$ Department of Genetics, University of Texas MD Anderson Cancer Center, Houston, Texas, USA. 'Division of Pulmonary Sciences and Critical Care Medicine, University of Colorado Denver School of Medicine, Aurora, Colorado, USA. ${ }^{7}$ College of Optometry, University of Houston, Houston, Texas, USA.

Airway mucin secretion is necessary for ciliary clearance of inhaled particles and pathogens but can be detrimental in pathologies such as asthma and cystic fibrosis. Exocytosis in mammals requires a Munc18 scaffolding protein, and airway secretory cells express all 3 Munc18 isoforms. Using conditional airway epithelial cell-deletant mice, we found that Munc18a has the major role in baseline mucin secretion, Munc18b has the major role in stimulated mucin secretion, and Munc18c does not function in mucin secretion. In an allergic asthma model, Munc18b deletion reduced airway mucus occlusion and airflow resistance. In a cystic fibrosis model, Munc18b deletion reduced airway mucus occlusion and emphysema. Munc18b deficiency in the airway epithelium did not result in any abnormalities of lung structure, particle clearance, inflammation, or bacterial infection. Our results show that regulated secretion in a polarized epithelial cell may involve more than one exocytic machine at the apical plasma membrane and that the protective roles of mucin secretion can be preserved while therapeutically targeting its pathologic roles.

Conflict of interest: MJT and BFD may work with their institution to file a patent based on the identification of a pharmacologic compound to inhibit stimulated mucin secretion as a therapeutic strategy to ameliorate airway mucus obstruction. BFD is a member of a scientific advisory board for Arrowhead Pharmaceuticals, which is using RNA interference to treat diseases affecting multiple organs, including mucus obstructive airway diseases, and his laboratory has received funding from Arrowhead Pharmaceuticals.

License: Copyright 2019, American Society for Clinical Investigation.

Submitted: September 11, 2018 Accepted: January 31, 2019 Published: March 21, 2019

Reference information: JCI Insight. 2019;4(6):e124815. https://doi.org/10.1172/jci. insight.124815.

\section{Introduction}

In mammalian conducting airways, mucus forms a critical barrier that protects the lungs from inhaled particles, pathogens, and toxicants (1). These foreign substances are trapped by mucus, which is swept out of the lungs by ciliary beating into the pharynx, where it is swallowed. Secreted polymeric mucins, the principal macromolecular components of mucus, are large, highly glycosylated proteins that polymerize into linear chains and networks $(2,3)$. Mucins are packaged dehydrated in secretory granules, and after exocytosis they interact with several hundred-fold their mass of water to expand and generate viscoelastic, gel-like mucus.

Two polymeric secreted mucins are expressed in the airway epithelium - Muc5b and Muc5ac. Mouse Muc5b is expressed constitutively in superficial epithelial cells and submucosal glands and is primarily responsible for mucociliary clearance. Deletion of the gene encoding Muc5b in mice results in death from bacterial infection and airway obstruction (4). Heterozygous gene deletion results in approximately $50 \%$ reduction in polystyrene bead clearance (5), showing that Muc5b is limiting for mucociliary clearance. Conversely, an overexpressing allele of human MUC5B is highly prevalent in people of European descent and shows evidence of positive selection, probably for its value in protection against lung infection even though it is a risk factor for pulmonary fibrosis late in life $(6,7)$. Muc5ac is expressed only at low levels in all airways of naive (uninflamed) mice and in distal airways of humans (1). However, Muc5ac expression rises approximately 40-fold during allergic inflammation $(8,9)$. Induced Muc5ac expression contributes importantly to helminth defense in the gut (10) and may help trap helminths migrating through the lungs (11). In allergic asthma, overexpression and rapid secretion of Muc5ac causes airway mucus occlusion and airflow obstruction (12). 
Mucins are secreted at a low baseline rate and a high agonist-stimulated rate $(13,14)$. Both rates are regulated by the second messengers diacylglycerol and calcium that act on the exocytic sensor Munc13-2 (15). Important extracellular agonists promoting baseline secretion are ATP and its metabolite adenosine, released predominantly from ciliated cells sensing shear stress from airflow during ventilation (14, $16,17)$. These agonists act on heptahelical receptors coupled by G proteins of the Gq subtype to PLC- $\beta$ that generates the second messengers diacylglycerol and inositol triphosphate, with the latter inducing the release of calcium from intracellular stores (13). Higher levels of the same agonists can stimulate high rates of mucin secretion (18), as can the neural and inflammatory mediators acetylcholine and histamine acting on the same pathway downstream of their cognate receptors (12). At high levels of intracellular calcium, the fast, low-affinity exocytic calcium sensor synaptotagmin 2 promotes mucin secretion (19). Baseline secretion is thought to be primarily responsible for clearance of inhaled particles and pathogens, while stimulated secretion can induce airway obstruction protectively to trap helminths or pathologically in asthma and other inflammatory airway disorders $(11,12)$.

Defects in mucin secretion in synaptosomal-associated protein 23-mutant (SNAP23-mutant) and vesicle-associated membrane protein 8-mutant (VAMP8-mutant) mice implicate the highly conserved SNARE (soluble $\underline{N}$-ethylmaleimide-sensitive factor attachment protein receptor) machinery in mucin exocytosis $(20,21)$. The SNARE complex is a 4-helix bundle comprising 3 helices attached to the target membrane ( $\mathrm{t}$-SNAREs) and 1 attached to the vesicle membrane (v-SNARE). Specific binding of these helices confers accuracy and directionality on the fusion reaction, and full coiling provides the energy to fuse the lipid membranes. SNARE-dependent vesicle traffic universally involves Sec1/ Munc18 (SM) proteins that promote SNARE complex assembly and help prevent off-target interactions (22). Yeasts contain 4 SM proteins, among which the exocytic protein Sec1 has evolved into 3 exocytic Munc18 isoforms in metazoans. We previously found, using heterozygous hypomorphic mutant mice (23), that Munc18b has a role in stimulated mucin secretion but were unable to identify a role in baseline secretion. Here, by performing a comprehensive analysis of airway epithelial deletion of all 3 Munc18 isoforms in mice, we sought to identify the Munc18 protein(s) mediating baseline secretion, to fully characterize the role of Munc18b in stimulated mucin secretion, and to test the hypothesis that selective impairment of stimulated secretion with preservation of baseline secretion can protect against airway mucus obstruction in pathophysiologic models.

\section{Results}

Generation of airway epithelial Munc18-deletant mice. We had previously found that homozygous hypomorphic mutant Munc18b mice are not viable after birth (23), and others similarly found that homozygous Munc18a-knockout (24) and Munc18c-knockout (25) mice are not viable. Therefore we assembled a panel of Munc18-conditional mutant mice by generating a conditional allele of Munc18b that is described here (Figure 1A and Supplemental Figure 1; supplemental material available online with this article; https://doi. org/10.1172/jci.insight.124815DS1), generating a conditional allele of Munc18c that is described elsewhere (26), and obtaining from Matthijs Verhage (University of Amsterdam, Amsterdam, the Netherlands) a conditional allele of Munc18a (27). Crossing conditional Munc18b mice with Zp3-Cre-Tg mice to generate whole animal-knockout mice did not yield any $\mathrm{Munc}^{18 \mathrm{~b}^{-/}}$pups, confirming that Munc18b is an essential gene in mice. Munc18b $\mathrm{b}^{-/-}$embryos grown in vitro developed to E3.5 at a Mendelian ratio, but in vivo, Munc18b ${ }^{-1-}$ embryos at E10.5 were present at less than a Mendelian ratio; at E11.5 there were none (Supplemental Table 1).

Deletion of Munc18b in airway epithelial cells by crossing Munc18b $\mathrm{b}^{\mathrm{F} / \mathrm{F}}$ mice with CCSP ${ }^{\mathrm{iCre}}$ mice (28) yielded Munc $18 \mathrm{~b}^{\mathrm{CCSP}-\Delta / \Delta}$ (hereafter Munc $18 \mathrm{~b}^{\Delta / \Delta}$ ) mice with normal litter sizes and weight at 3 weeks compared with Munc $18 \mathrm{~b}^{\mathrm{F} / \mathrm{F}}$ mice (Figure 1, B and C). The efficiency of recombination of CCSP $^{i \text { Cre }}$ mice at the ROSA26 locus is greater than $99 \%$ in both airway secretory and ciliated cells (Supplemental Figure 2, A and B), and recombination occurs sporadically in alveolar type 2 secretory epithelial cells as well (Supplemental Figure 2C). Histopathologically, the lungs of all floxed and single Munc18 isoform airway-deletant mice were unremarkable upon H\&E staining and upon immunohistochemical staining for numbers and morphology of $\mathrm{CCSP}^{+}$secretory cells and AcTub ${ }^{+}$ciliated cells (Figure 2), as were the lungs of Munc18a/b- and Munc18b/c-floxed mice and Munc18a/b-double deletant mice (Supplemental Figure 3, A and B). However, the airways of Munc18b/c-double deletant mice showed a flattened epithelium with an almost complete absence of airway secretory cells upon immunohistochemical staining for CCSP (Supplemental Figure 3C), and the lung alveolar regions 
A
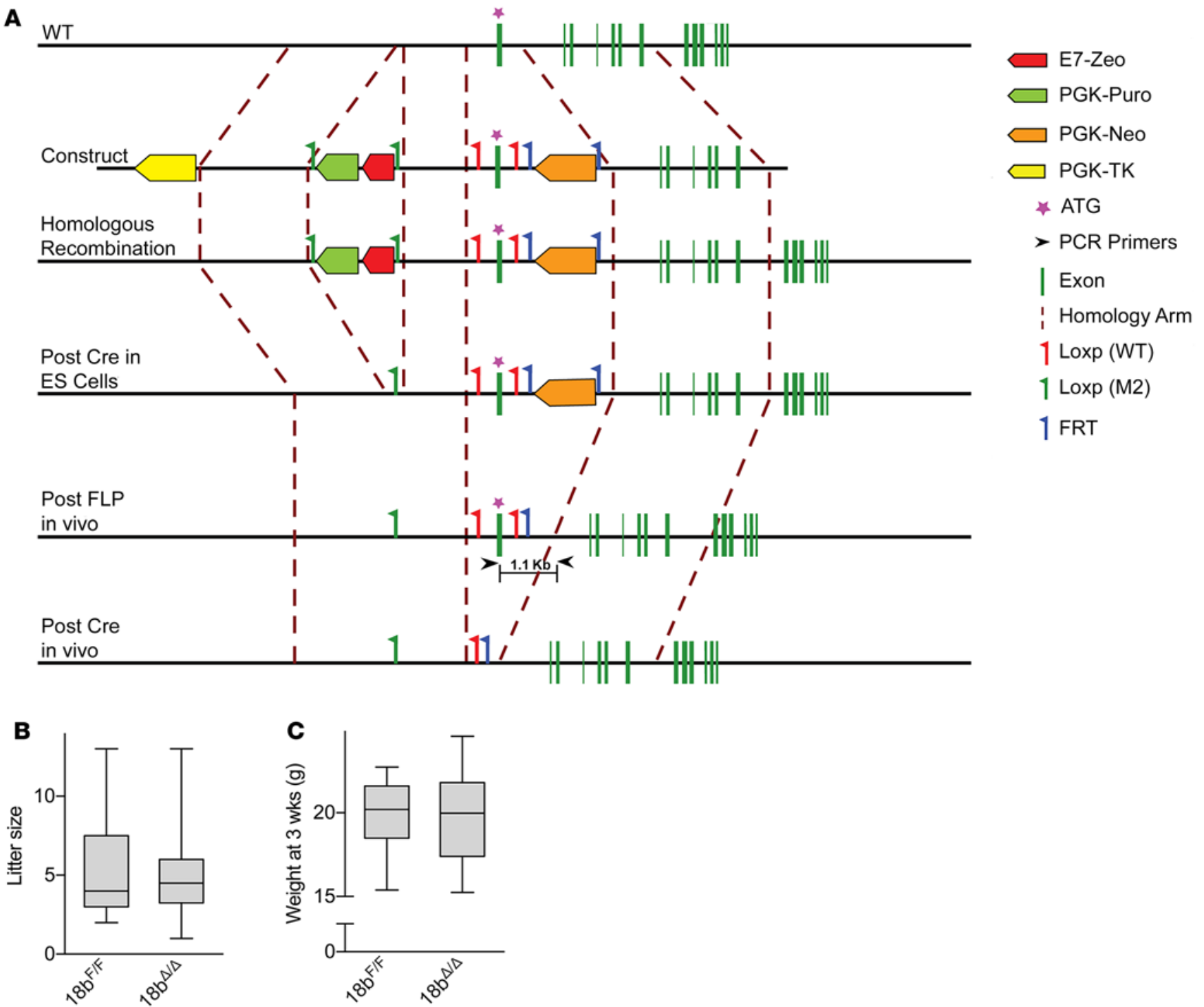

Figure 1. Generation of Munc18b-conditional deletant mice. (A) Exon 1 of the Munc18b gene was flanked by 2 loxP sites (red flags) via homologous recombination. Herpes simplex virus thymidine kinase (PGK-TK, shown in yellow) was used for negative selection. Zeocin (e7-Zeo, shown in red) and puromycin resistance genes (PGK-Puro, shown in green) flanked by 2 loxP (M2) sites (green flags), removed by Cre in embryonic stem cells, and a neomycin resistance gene (PGK-Neo, shown in orange) flanked by 2 Flp recognition target (FRT) sites (blue flags), removed by Flp recombination, were used for positive selection. Exon 1 was removed by Cre recombination in mice. ATC, transcriptional start site; ES, embryonic stem. (B) Litter size after crossing a floxed mouse in $\mathbf{A}$ to a CCSPicre mouse to generate a conditional deletant mouse specific for airway epithelium are compared with their floxed littermates ( $n=50-52$ per group). (C) Weight at 3 weeks of age of Munc18b-conditional deletant mice and their floxed littermates ( $n=50-52$ per group). In box plots, line represents median; box, 25th-75th percentile; and whiskers, 5th-95th percentile for this and all subsequent figures.

showed emphysema (Supplemental Figure 3D). To determine whether these abnormalities reflected dependency on Munc18b and Munc18c only during development, we used CCSPCreER mice to induce recombination during adulthood (29). Airway secretory cell viability was still impaired because CCSP expression was lost 2 weeks after recombination (Supplemental Figure 3E), though emphysema was no longer present (Supplemental Figure 3F).

To determine the normal expression of Munc18 isoforms in the airway epithelium and confirm the efficiency of gene deletion, we performed quantitative in situ hybridization with riboprobes. Munc18a and Munc18b transcripts were expressed in secretory cells at levels several-fold higher than in ciliated cells (Figure 2, A and B), whereas Munc18c transcripts were expressed in both cell types at similar levels (Figure 2C). All 3 Munc18-conditional deletant mice showed no significant expression of cognate transcripts 
A CCSP AcTub DAPI riboprobe
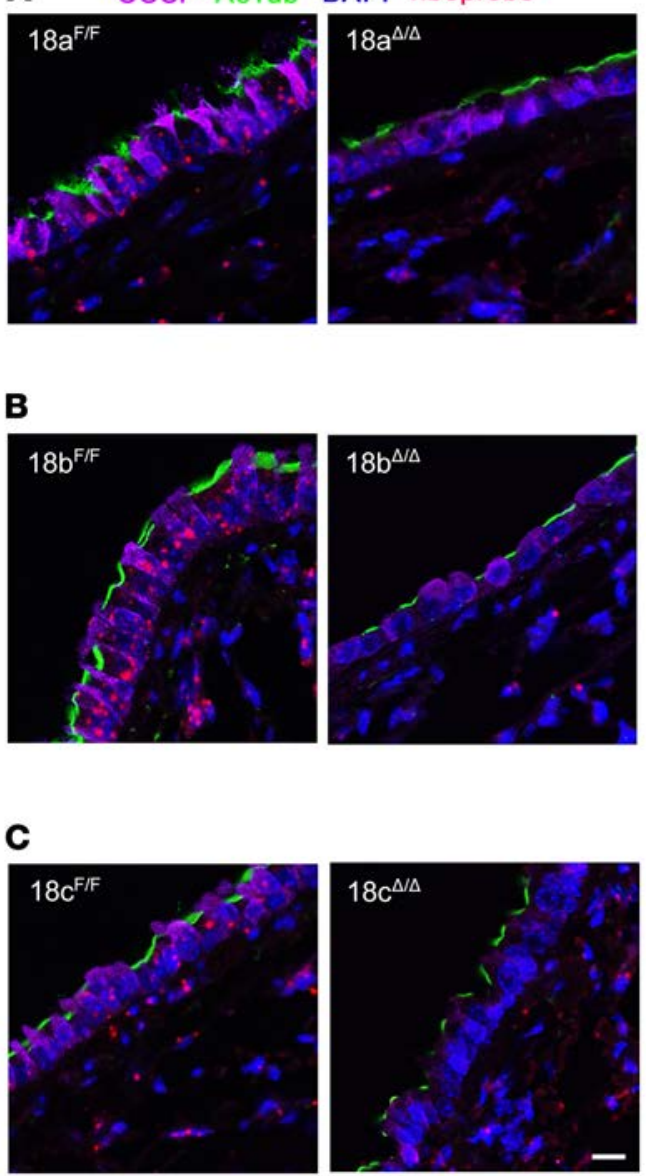
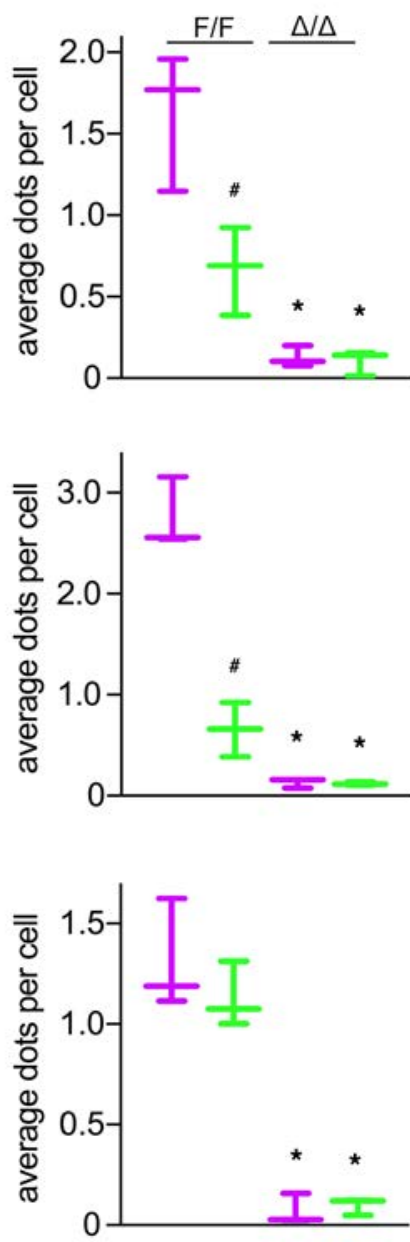
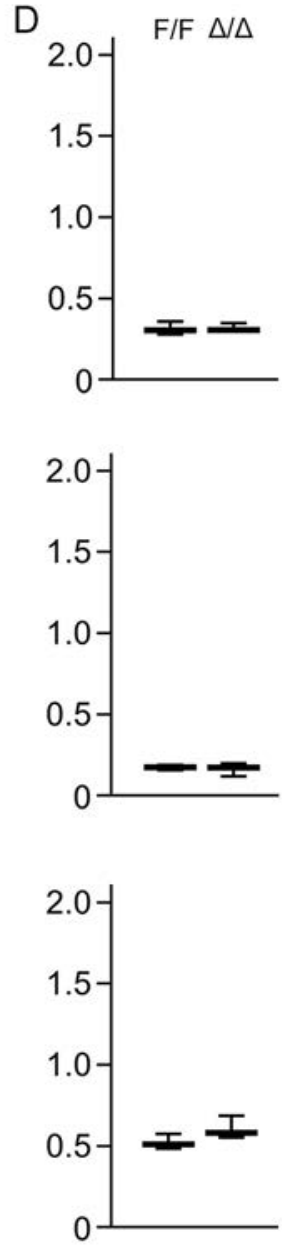

Figure 2. In situ hybridization of Munc18 isoforms. Representative images of in situ hybridization with fluorescent-labeled riboprobes (shown in red), specific for murine Munc18a (A), Munc18b (B), and Munc18c (C). Sections are from naive (uninflamed) lungs of floxed and conditional deletant mice. CCSP was used as a secretory cell marker (shown in purple) and acetylated tubulin as a ciliated cell marker (shown in green). Graphs show quantification of dots per cell type, per genotype, for each probe. (D) Quantification of riboprobes in the subepithelial tissue. Scale bar: $30 \mu \mathrm{m}$ ( $n=3$ mice per group). (A) Secretory versus ciliated $\left(18 a^{\mathrm{F} / F}\right), P=0.03$; secretory $\left(18 a^{\mathrm{F} / F}\right)$ versus secretory $\left(18 a^{\Delta / \Delta}\right), P=0.0038$; ciliated $\left(18 a^{\mathrm{F} / F}\right)$ versus ciliated $\left(18 \mathrm{a}^{\Delta / \Delta}\right), P=0.0254 .(\mathrm{B})$ Secretory versus ciliated $\left(18 \mathrm{~b}^{\mathrm{F} / F}\right), P=0.0012$; secretory $\left(18 \mathrm{~b}^{\mathrm{F} / F}\right)$ versus secretory $\left(18 \mathrm{~b}^{\Delta / \Delta}\right), P=0.0002$; ciliated $\left(18 \mathrm{~b}^{\mathrm{F} / \mathrm{F}}\right)$ versus ciliated $\left(18 \mathrm{~b}^{\Delta / \Delta}\right), P=0.0253 .(\mathrm{C})$ Secretory $\left(18 \mathrm{c}^{\mathrm{F} / \mathrm{F}}\right)$ versus secretory $\left(18 \mathrm{c}^{\Delta / \Delta}\right), P=0.0017$; ciliated $\left(18 \mathrm{c}^{\mathrm{F} / \mathrm{F}}\right)$ versus ciliated $\left(18 \mathrm{c}^{\Delta / \Delta}\right), P=0.0004$, Student's 2 -tailed $t$ test. ${ }^{\#} P=0.05$ between cell types; ${ }^{*} P=0.05$ within cell type.

(Figure 2), and there was no significant difference in transcript expression between any of the floxed mice and WT mice (not shown). Furthermore, transcript expression in subepithelial tissue was low and similar between Munc18-floxed and -deletant mice (Figure 2D), demonstrating the strength of expression of Munc18 isoforms in epithelial secretory cells and the cellular specificity of deletion.

Munc18b predominates in stimulated mucin secretion. Our previous study using heterozygous mutant mice indicated that Munc18b has a major role in stimulated mucin secretion (23). To comprehensively analyze Munc18 function in stimulated secretion, mucin production was first increased (mucous metaplasia) in all mutant mice using ovalbumin (OVA) sensitization and challenge to induce allergic inflammation, and then mice were stimulated with the secretagogue ATP to induce secretion acutely (Figure 3A). None of the Munc18-floxed mice showed a phenotype in mucous metaplasia or in mucin secretion in this or any subsequent experiments (Supplemental Figure 1B). All 3 Munc18 single-conditional deletant mice had mucin content similar to WT mice after the OVA challenge, but the Munc18a/b-double deletant mice had significantly higher mucin content (Figure 3, A and B), suggesting a defect in baseline secretion that results in mucin accumulation (see below). After ATP exposure, the predominant role of Munc18b was confirmed because the conditional deletant mice secreted only approximately $26 \%$ of intracellular mucin (mean value) compared with Munc18b-floxed mice, who secreted approximately $60 \%$, or WT mice, who 
A
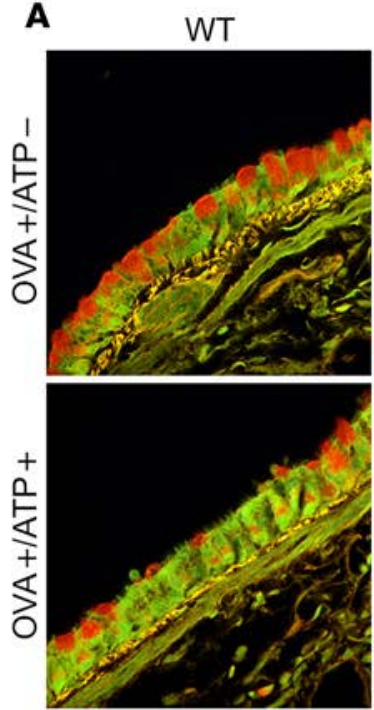

B

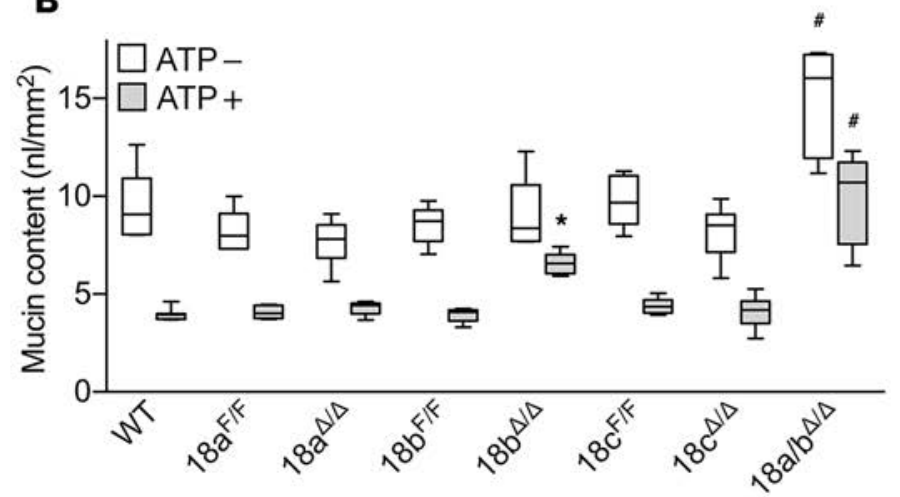

$18 \mathrm{~b}^{\Delta / \Delta}$
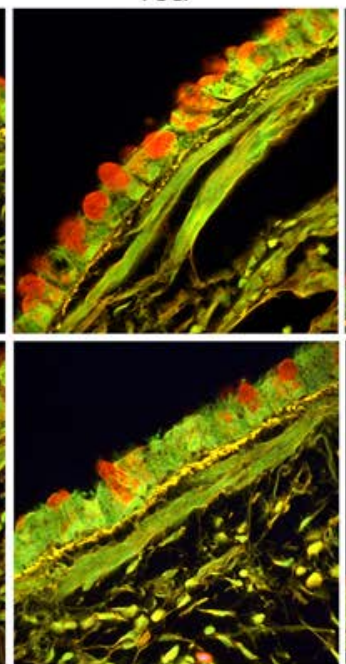
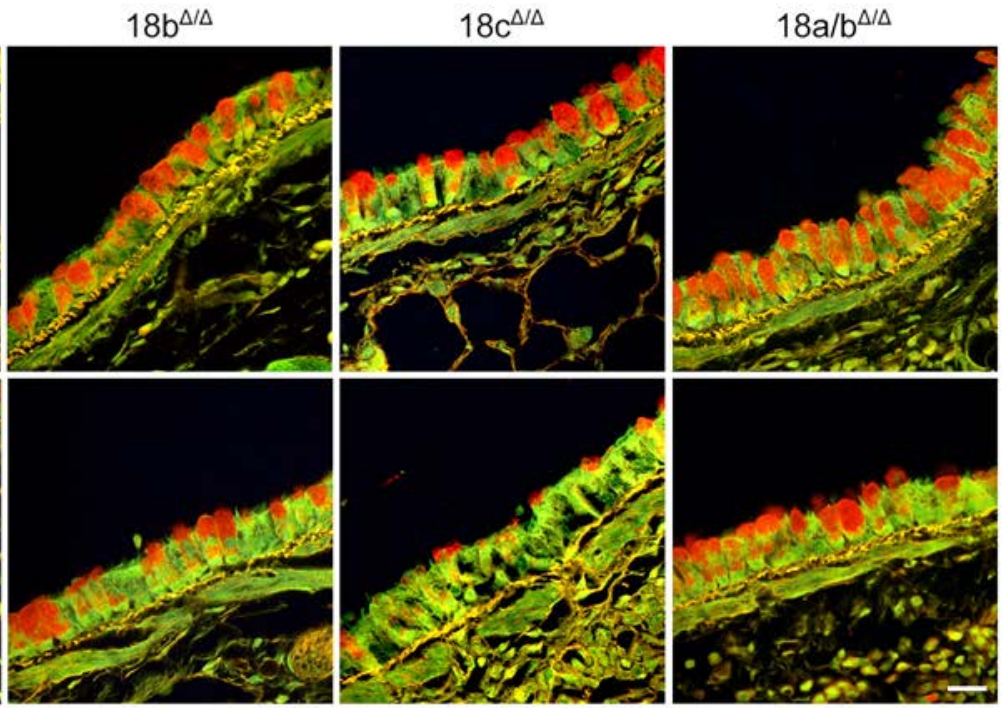

C

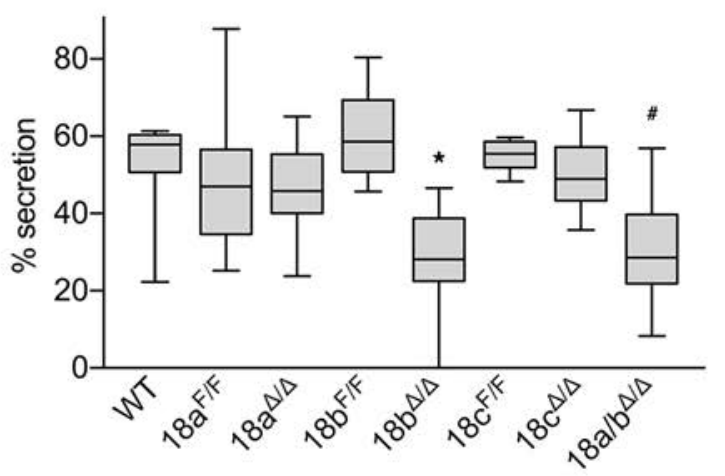

Figure 3. Stimulated mucin secretion measured by residual intracellular mucin content. (A) High-magnification views of representative fields of PAFSstained bronchial airways from mice sensitized and challenged with OVA to increase mucin production (OVA+/ATP-, top row), then exposed to aerosolized $100 \mathrm{mM}$ ATP to stimulate mucin secretion (OVA+/ATP+, bottom row). Scale bar: $20 \mu \mathrm{m}$. (B) Quantification of the volume density (expressed as nl mucin per $\mathrm{mm}^{2}$ basement membrane) of intracellular mucin in mice with or without ATP stimulation as in $\mathbf{A}$ (representative experiment of 3 separate experiments with all genotypes, $n=5-8$ mice per group). 18a/b $b^{\Delta / \Delta}$ (ATP-) versus WT (ATP-), $P<0.0001$, versus 18a $a^{F / F}$ (ATP-), P<0.0001, versus 18b $\mathrm{b}^{\mathrm{F} / \mathrm{F}}$ (ATP-), $P<$ 0.0001 , Tukey's test. 18b $\mathrm{b}^{\Delta / \Delta}\left(\right.$ ATP + ) versus $18 \mathrm{~b}^{\mathrm{F} / \mathrm{F}}(\mathrm{ATP}+), P<0.0001 ; 18 \mathrm{a} / \mathrm{b}^{\Delta / \Delta}(\mathrm{ATP}+)$ versus WT $(\mathrm{ATP}+), P<0.0001$, versus $18 \mathrm{a}^{\mathrm{F} / \mathrm{F}}(\mathrm{ATP}+), P=0.0001$, versus $18 b^{F / F}(A T P+), P<0.0001$, Student's 2-tailed $t$ test. (C) The percentage of mucin released for each genotype ( 3 independent experiments like those in B, combined). $18 \mathrm{~b}^{\Delta / \Delta}$ versus $18 \mathrm{~b}^{\mathrm{F} / \mathrm{F}}, P<0.0001 ; 18 \mathrm{a} / \mathrm{b}^{\Delta / \Delta}$ versus $\mathrm{WT}, P=0.001$, versus $18 \mathrm{a}^{\mathrm{F} / \mathrm{F}}, P<0.0001$, versus $18 \mathrm{~b}^{\mathrm{F} / \mathrm{F}}, P<0.0001$, Mann-Whitney test. ${ }^{*} P<$ 0.05 versus floxed littermate; ${ }^{\#} P<0.05$ versus WT.

secreted approximately 52\% (Figure 3C). Munc18a- and Munc18c-deletant mice secreted as efficiently as their cognate floxed mice or WT mice, while the Munc18a/b-double deletant mice secreted approximately $31 \%$, comparable to the Munc18b-single deletant mice (Figure 3C).

Munc18a predominates in baseline mucin secretion. No defect in baseline mucin secretion had been observed in Munc18b heterozygous mutant mice (23). This could be due either to the actual lack of a role of Munc18b or to the lack of an obvious phenotype with just a 50\% reduction in protein expression. To further study baseline mucin secretion, all Munc18 airway-deletant mice were examined.

In naive (uninflamed) WT mice, the rate of mucin secretion closely matches the rate of mucin production such that intracellular mucin does not accumulate and is not visible by periodic acid fluorescent Schiff reagent (PAFS) histochemical staining. Hence, a defect in baseline mucin secretion can be detected as spontaneous mucin accumulation $(15,30)$. Munc18a-deletant mice showed significant spontaneous mucin accumulation, indicating a role of Munc18a in baseline secretion (Figure 4, A and B). Munc18b- and Munc18c-deletant mice showed no apparent mucin accumulation. However, Munc18a/b-double deletant mice showed a higher level of mucin accumulation than Munc18a-single deletant mice, indicating an addi- 
A
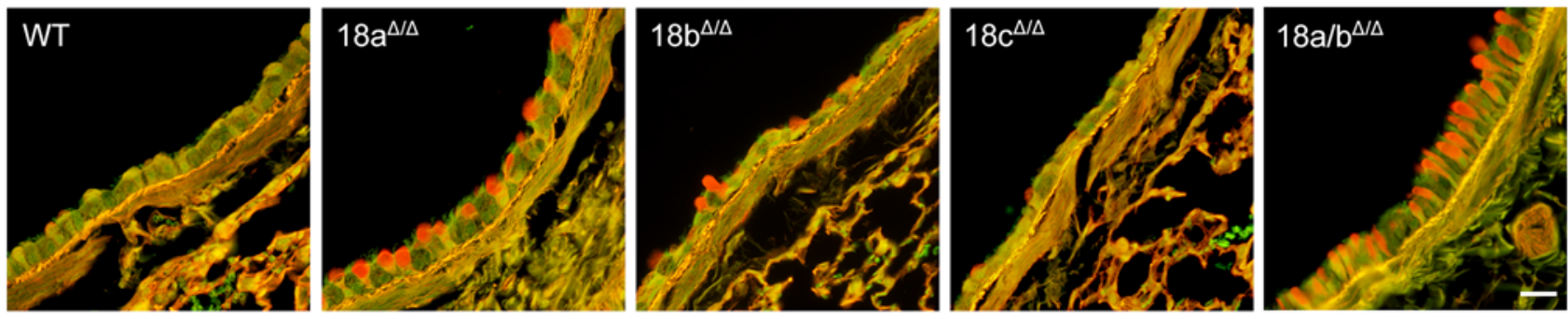

B

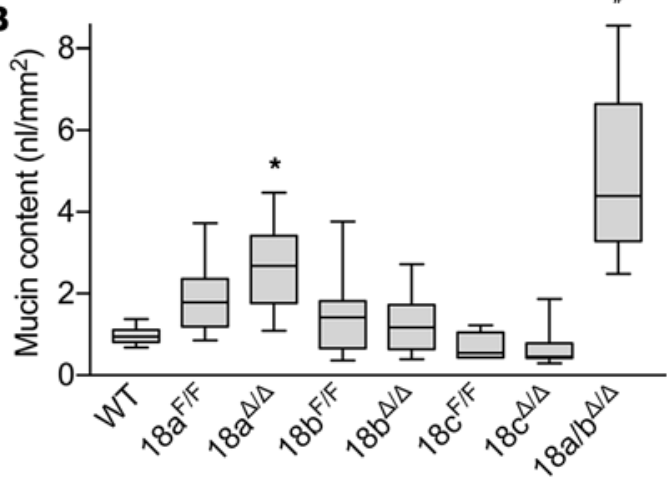

C

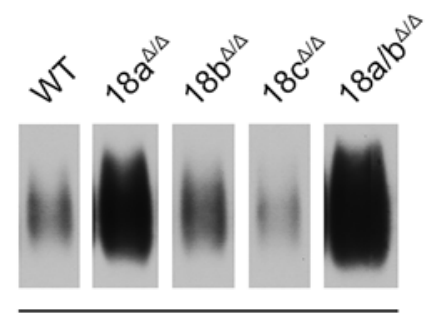

Muc5b

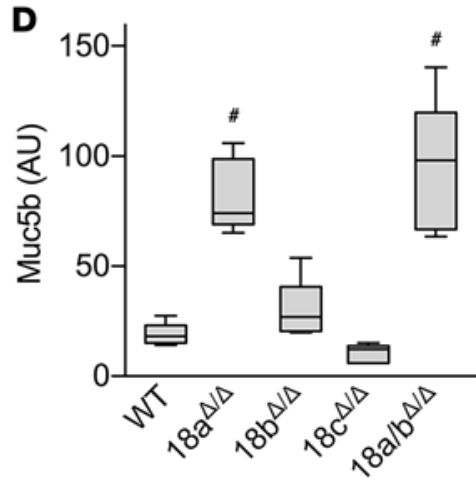

Figure 4. Baseline mucin secretion measured by spontaneous intracellular mucin accumulation. (A) High-magnification views of representative fields of PAFS-stained bronchial airways from naive mice. Scale bar: $20 \mathrm{~m}$. (B) Quantification of the volume density of spontaneous intracellular mucin accumulation ( $n=5-18$ mice per group, 3 independent experiments combined). $18 a^{\Delta / \Delta}$ versus $18 a^{\mathrm{F} / \mathrm{F}}, P=0.0243 ; 18 \mathrm{a} / \mathrm{b}^{\Delta / \Delta}$ versus $\mathrm{WT}, P<0.0001$, versus $18 \mathrm{a}^{\Delta / \Delta}$, $P=0.0008$, Student's 2-tailed $t$ test. (C) Representative immunoblot of $50 \mu \mathrm{g}$ of whole-lung lysates from naive mice probed for Muc5b. (D) Densitometric analysis of immunoblot shown in C derived from standard curve (Supplemental Figure 4$)\left(n=5-7\right.$ mice per group). AU, arbitrary units. $18 a^{\Delta / \Delta}$ versus WT, $P=0.0079 ; 18 \mathrm{a} / \mathrm{b}^{\Delta / \Delta}$ versus WT, $P=0.0025$, Mann-Whitney test. ${ }^{*} P<0.05$ versus floxed littermate; ${ }^{\#} P<0.05$ versus $W T$.

tive effect of Munc18a and Munc18b in baseline mucin secretion. Spontaneous mucin accumulation was further analyzed by quantitative immunoblotting for Muc5b, which is expressed in the airways of naive mice $(4,8,15,31)$. This confirmed significant mucin accumulation in Munc18a-deletant mice $(P=0.007)$, a trend toward a small degree of accumulation in Munc18b-deletant mice $(P=0.09)$, and an additive effect in Munc18a/b-double deletant mice (Figure 4, C and D, and Supplemental Figure 4). Muc5ac is not expressed significantly in the airways of naive mice $(4,8,31)$ and was not detected in immunoblots of the lungs of any naive Munc18-deletant mice (not shown).

To rule out the possibility that spontaneous mucin accumulation was due to an increase in mucin expression resulting from an inflammatory response in any of the deletant mice, mRNA expression was analyzed by quantitative reverse transcription PCR in Munc18-single deletant and Munc18a/b-double deletant mice. There was no significant increase in expression of $M u c 5 a c$ or $M u c 5 b$ in any of the Munc18-deletant mice (Supplemental Figure 6A). To evaluate whether partial Munc18-single deletant phenotypes might be due to compensation from upregulation of the expression of another isoform, transcripts of all 3 Munc18 isoforms were measured in the single-deletant mice. There was no upregulation of another Munc18 isoform upon deletion of any of the Munc18 genes (Supplemental Figure 6B). Further, there was no upregulation of any Munc18 transcript during mucous metaplasia (Supplemental Figure 6B), consistent with our prior finding that Munc18b protein level does not change during mucous metaplasia (23).

To examine granule morphology and number by electron microscopy, mice were lightly stimulated with $\mathrm{IL}-13$ so that mucin granules were readily visible (Supplemental Figure 5A). Munc18a/b-double deletant mice showed an increase in surface-to-volume density of granules (SvG) by stereology (Supplemental Figure 5B), indicating smaller granules with a lower ratio of surface area to volume, though neither Munc18-single deletant phenotype differed from WT. Both Munc18a- and Munc18b-single deletant mice showed an increased number of granules, and Munc18a/b-double deletant mice showed a further increase (Supplemental Figure 5C). In addition, mucin granules in Munc18a/b-double deletant mice, and to lesser extents those in Munc18a- and Munc18b-single deletant mice, were found to be more electron dense (Supplemental Figure 5D). 
Deletion of Munc18a or Munc18b does not impair mucociliary clearance. The absence of an increase in mucin transcripts in the Munc18 airway-deletant mice suggested that mucociliary clearance function was preserved, preventing lung infection and inflammation that might induce mucin gene upregulation (Supplemental Figure 6A). To further test these inferences, several additional studies were performed. First, lung lavage fluid was obtained for measurement of leukocytes, which is a sensitive indicator of inflammatory status. There was no difference in total lavage cell number or fractional representation of any leukocyte subset in either of the Munc18a- or Munc18b-single deletant mice compared with WT or floxed littermate mice (Supplemental Figure 6C). However, Munc18a/b-double deletant mice showed a small increase in neutrophil number, suggesting either a small defect in mucociliary clearance not detected by our assay or a small increase in epithelial pyroptosis resulting from mild impairment of epithelial viability. Next, polystyrene beads were instilled into the lungs to measure their clearance by mucociliary transport. There was no difference in the fraction of beads cleared by Munc18a- or Munc18b-deletant mice compared with WT (Supplemental Figure 6D). Last, the lung microbiome was interrogated by quantitative PCR (qPCR) and sequencing of 16S ribosomal RNA. There was no significant difference in the quantity (Supplemental Figure 6E) or composition (Supplemental Figure 6F) of bacteria present in the lungs of Munc18-single deletant or Munc18-double deletant mice compared with WT or floxed littermate mice.

Deletion of Munc18b protects mice against airway obstruction in a model of allergic asthma. Increased mucin production followed by stimulated secretion causes airway lumenal mucus occlusion and airflow obstruction in asthma $(1,12)$. We hypothesized that deletion of Munc18b in airway secretory cells would protect against this pathophysiology. To test this, we first performed a pilot study in a WT mouse with IL-13 instilled intrapharyngeally to induce mucous metaplasia and then exposed to a methacholine aerosol to stimulate mucin secretion. We measured the occlusion of airways throughout the lungs at $500-\mu \mathrm{m}$ intervals as a fraction of cross-sectional airway lumenal area and found that the right caudal lobe had the highest fractional occlusion (Supplemental Figure 7A). We next compared fractional occlusion in the right caudal lobe between Munc18b-floxed and -deletant mice and found a significant reduction ( $\sim 50 \%$, mean values) in the Munc18b-deletant mice (Supplemental Figure $7 \mathrm{~B})$. We then performed a definitive study comparing the sum of the area of lumenal mucus in the right caudal lobe at 1-mm intervals (Figure 5A) together with measurement of lung mechanics. Munc18b-deletant mice showed an approximately $62 \%$ reduction (mean values) in lumenal mucus area compared with Munc18b-floxed mice, similar to the approximately 37\% reduction in Muc5ac-knockout mice, whereas Munc18a-deletant mice showed no reduction (Figure 5B).

Exposure to methacholine induces resistance to airflow because of a combination of smooth muscle contraction (bronchoconstriction) and mucus obstruction (12). An augmented response to methacholine (airway hyperresponsiveness) is a sensitive indicator of asthmatic airway dysfunction. WT mice with mucous metaplasia induced by IL-13 who were then exposed to increasing concentrations of aerosolized methacholine showed increased respiratory system resistance compared to naive mice

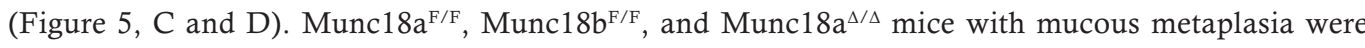
similar to WT mice, but Munc18b $\mathrm{b}^{\Delta / \Delta}$ and $\mathrm{Muc}^{\mathrm{a}} \mathrm{ac}^{-/-}$mice with mucous metaplasia were highly protected from airway hyperresponsiveness to methacholine (Figure 5, C and D).

Deletion of Munc18b protects mice against airway mucus occlusion and parenchymal emphysema in a model of cystic fibrosis. In cystic fibrosis, an inherited defect in transepithelial anion and water transport causes the formation of mucus that is excessively concentrated, viscoelastic, and adhesive (32-34). Mucus accumulates because its excessive viscoelasticity impedes clearance by ciliary beating, and its adhesiveness results in the formation of mucus plaques. These, in turn, lead to infection and inflammation that cause progressive lung disease. In mice, deletion of the anion transporter cystic fibrosis transmembrane conductance regulator (CFTR) does not result in lung disease because of the presence of alternative mechanisms of anion transport, but Tg overexpression of the $\beta$ subunit of the epithelial $\mathrm{Na}^{+}$channel (ENaC) results in concentrated mucus, leading to lung disease that resembles human cystic fibrosis (35).

To test whether stimulated mucin secretion contributes to pathophysiology in this model, we crossed Munc18b-deletant mice with $\beta$-ENaC-Tg mice (Figure 6A). Mucus occlusion in $\beta$-ENaC-Tg mice was reduced by approximately $66 \%$ (mean values) by deletion of Munc18b in the airway (Figure $6 \mathrm{~B})$. Emphysema measured as equivalent mean diameter $\left(\mathrm{D}_{2}\right)$ was increased approximately $86 \%$ in $\beta$-ENaC-Tg Munc18b $\mathrm{b}^{\mathrm{F} / \mathrm{F}}$ mice compared with Munc18 $8^{\mathrm{F} / \mathrm{F}}$ mice, as previously described for $\beta$-ENaC- 
A

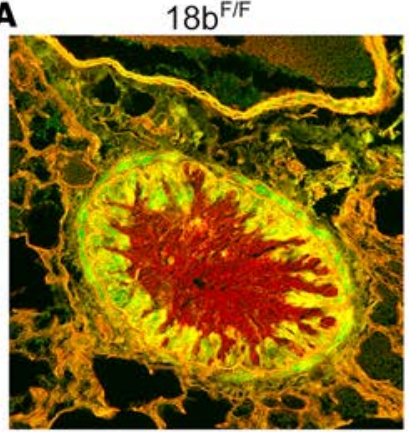

B

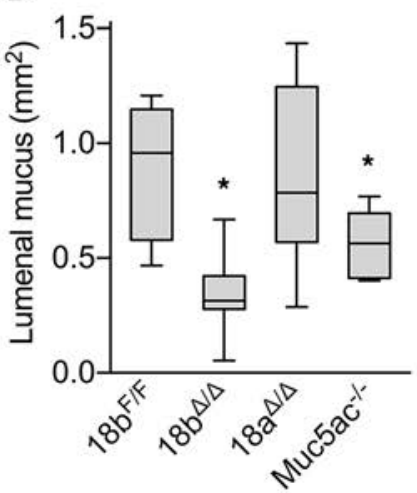

$18 b^{\Delta / \Delta}$

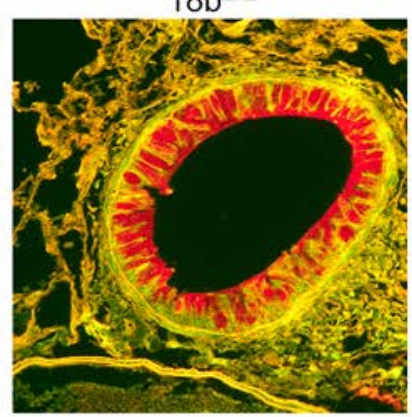

C

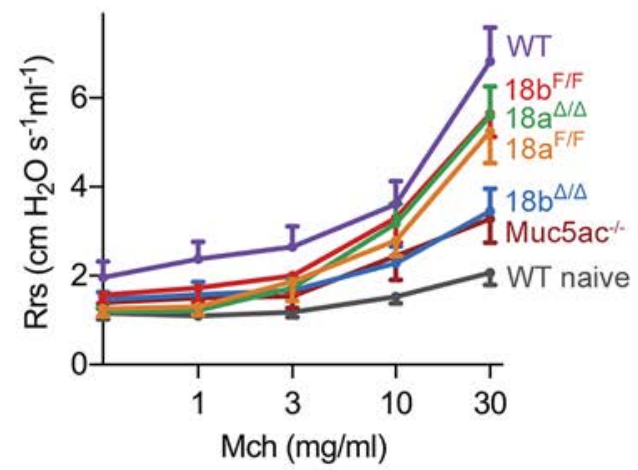

$18 a^{\Delta / \Delta}$
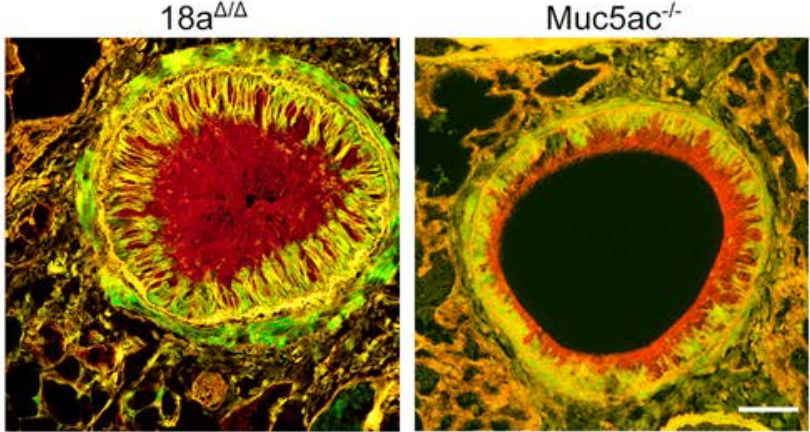

D

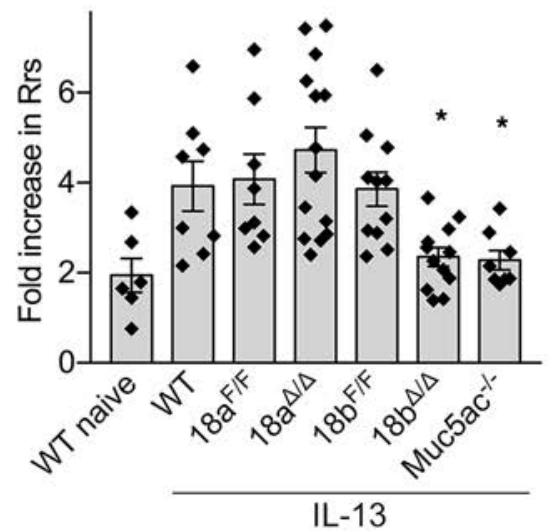

Figure 5. Airway mucus occlusion and airway hyperreactivity of Munc18b-conditional deletant mice in an allergic asthma model. (A) Representative cross-sections of airways fixed with methacarn to preserve mucus volume and stained with PAFS. Mice were treated with IL-13 to induce mucin production and then stimulated with aerosolized $150 \mathrm{mM}$ methacholine (Mch) to stimulate mucin secretion and smooth muscle contraction. Scale bar: $50 \mu \mathrm{m}$. (B) Cross-sectional area of lumenal mucus in the right caudal lobe measured at 1,000- $\mu \mathrm{m}$ intervals $(n=5-9$ mice per group, representative experiment, $>100$ airways per group quantified). $18 \mathrm{~b}^{\Delta / \Delta}$ versus $18 \mathrm{~b}^{\mathrm{F} / \mathrm{F}}, P=0.0002$; Muc5ac ${ }^{-/-}$versus $18 \mathrm{~b}^{\mathrm{F} / \mathrm{F}}, P=0.0378$, Student's 2 -tailed $t$ test. (C) Total respiratory system resistance (Rrs) at increasing doses of nebulized Mch in mice treated with or without IL-13 ( $n=6-14$ per group, 3 independent experiments combined). Line represents mean; error bar, SEM. (D) Fold-change in Rrs at the highest dose of nebulized Mch (30 mg/ml) from C.

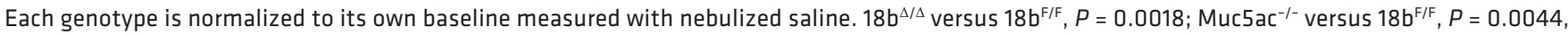
Student's 2-tailed $t$ test. ${ }^{*} P<0.05$ versus floxed littermate. Bar, mean; error bar, SEM.

Tg mice (ref. 35 and Figure 6C). This increase was attenuated by approximately $22 \%$ by deletion of Munc18b in the airway (Figure 6C). However, lung neutrophilic and eosinophilic inflammation present in $\beta$-ENaC-Tg Munc18b $b^{\mathrm{F} / \mathrm{F}}$ mice was not reduced by Munc18b deletion (Figure 6D).

\section{Discussion}

Here, we have performed a comprehensive analysis of the function of Munc18 proteins in a polarized epithelial cell specialized for apically directed, regulated secretion. Our central finding is that baseline and stimulated secretion are predominantly mediated by different Munc18 proteins, with Munc18a having the major role in baseline secretion, Munc18b having the major role in stimulated secretion, and Munc18c having no apparent role. This finding has implications for understanding the cell biology of regulated secretion in polarized cells and for manipulating the exocytic machinery of the airway epithelium therapeutically to alleviate mucus dysfunction.

Regarding cell biology, Munc18b has been described previously by us and others as mediating apical secretion in polarized epithelial cells $(23,36,37)$. Munc18a has been studied primarily for its role in synaptic vesicle release from neurons $(24,38,39)$, but other regulated exocytic systems where Munc18a has been reported to function include vascular endothelial cells (40) and acrosomal exocytosis in spermatozoa (41). In airway epithelium, Munc18a was reported to modulate the conductance of the apical anion channel CFTR (42), but a role in vesicular transport was not described. Munc18a and Munc18b have been reported to cooperate in regulated exocytosis of insulin-containing granules of pancreatic islet cells $(43,44)$ 
A

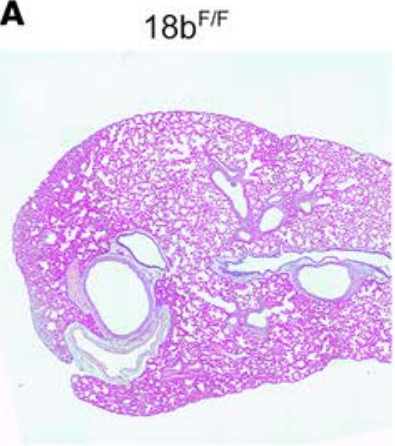

$18 b^{\Delta / \Delta}$

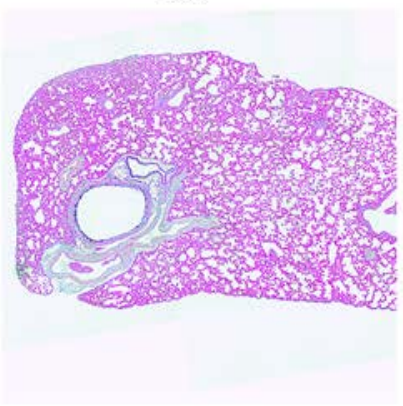

$\mathrm{ENaC}-18 \mathrm{~b}^{\mathrm{F} / \mathrm{F}}$

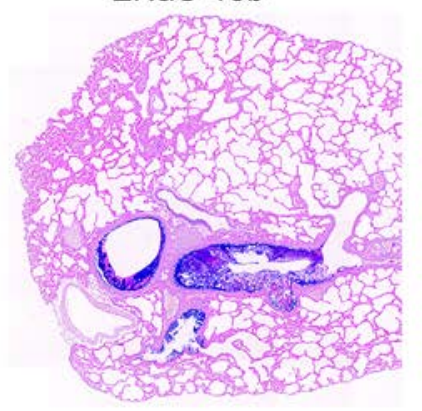

$\mathrm{ENaC}-18 b^{\Delta / \Delta}$

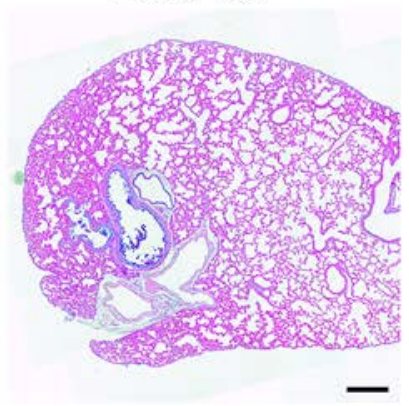

B

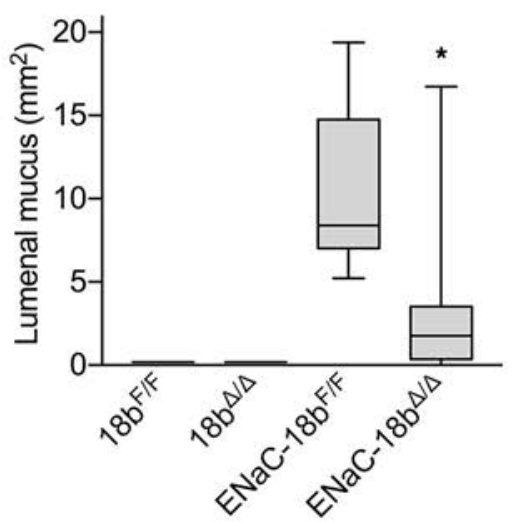

C

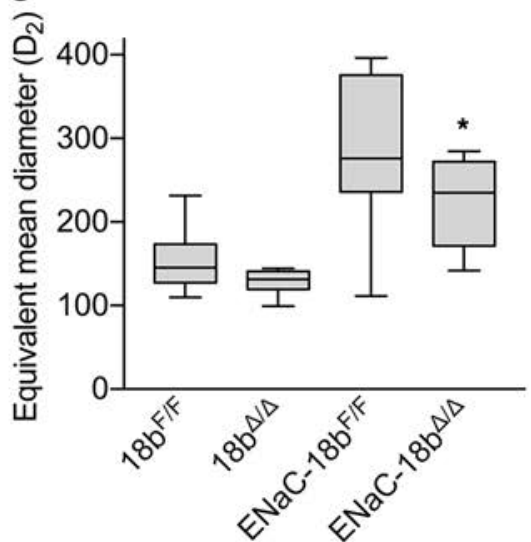

D

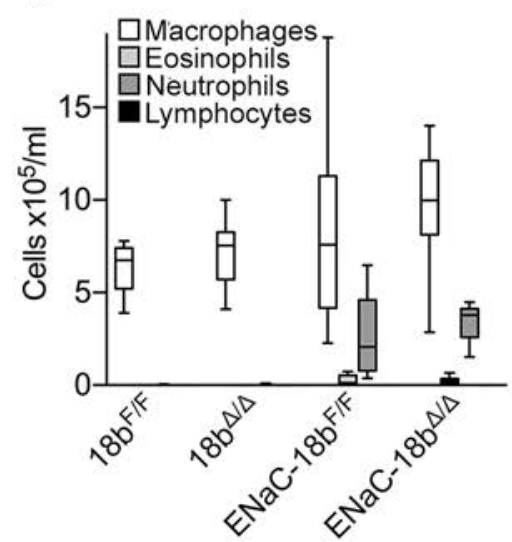

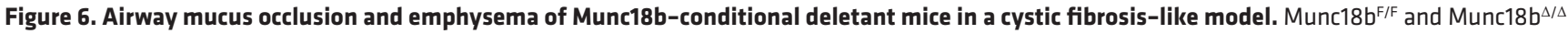
mice were crossed or not to $\beta$-ENaC-overexpressing-Tg mice. (A) Representative transverse left lung sections stained with Alcian blue and periodic acid-

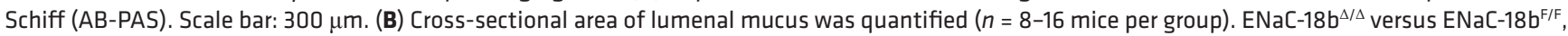
$P=0.0013$, Mann-Whitney test. (C) Emphysema was assessed as the $D_{2}\left(n=8-16\right.$ mice per group). ENaC-18b $\mathrm{b}^{\Delta / \Delta}$ versus ENaC $-18 \mathrm{~b}^{\mathrm{F} / \mathrm{F}}, P=0.0009, \mathrm{Student}$ 's 2-tailed $t$ test. (D) Total inflammatory cell numbers from lung lavage fluid ( $n=8-16$ mice per group). ${ }^{*} P<0.05$ versus floxed littermate.

and cytolytic granules of natural killer cells (45) in response to different signaling pathways. Prior reports that Munc18a and Munc18b both participate in mast cell degranulation appear to have been in error, with Munc18b having an exclusive role as we recently described (26). Thus, the current work is the first report, of which we are aware, of 2 Munc18 proteins mediating different rates of secretion in response to the same signaling pathway at a single polarized epithelial surface.

Munc18c has been proposed to mediate basolateral secretion in polarized epithelial cells $(46,47)$, consistent with the lack of effect of Munc18c deletion in apical regulated secretion in our system (Figures 3 And 4). A role for Munc18c in stimulated exocytosis has been described in nonpolarized cells, such as translocation of glucose transporters in adipocytes (48), but not in polarized epithelia. The cellular lethality induced by simultaneous deletion of both Munc18b and Munc18c in airway epithelial cells (Supplemental Figure 3) suggests that the predominant function of Munc18c is in constitutive secretion because that is an essential cellular function. We hypothesize that Munc18c function is rescued in the single airway-deletant mouse by ectopic function of Munc18b. This hypothesis is consistent with the cellular viability of airway epithelial cells in Munc18a/b-double deletant mice (Figures 3 And 4) because regulated secretion is not a cell-autonomous, essential function. Whether Munc18c mediates constitutive secretion at both the apical and basolateral surfaces in airway epithelial cells or functions exclusively at the basolateral surface is not known.

It is notable that the phenotypes of Munc $18 \mathrm{a} / \mathrm{b}$-double deletant mice are severer than those of Mun$\mathrm{c} 18 \mathrm{a}-$ and Munc18b-single deletant mice in baseline and stimulated secretion, respectively (Figures 3 and 4). Furthermore, even the Munc18a/b-double deletant mice do not have complete defects in baseline secretion, as evidenced by the ability of secretory cells to further accumulate mucin when production is stimulated by IL-13 (compare Figures 3 And 4), or in stimulated secretion, as evidenced by residual ATP-stimulated mucin release (Figure 3). Measurement of Munc18 transcripts in deletant mice rules out upregulation of the expression of other isoforms as a possible mechanism of compensation (Supplemental 
Figure 6B). Moreover, it is unclear whether the exocytic pathways mediated by Munc18a and Munc18b overlap partially such that mucins can take an alternative pathway when the normal route is unavailable or whether noncognate Munc18 proteins can function ectopically to a small degree when the cognate protein is not available. We favor the latter explanation, with even the more distantly related Munc18c protein able to partially rescue function in the Munc18a/b-double deletant mice.

Because Munc18 proteins partner with specific syntaxins t-SNAREs (23), our findings here suggest that the exocytic SM-SNARE machinery is mostly different between baseline and stimulated mucin secretion. This inference is supported by the role of the v-SNARE VAMP8 predominantly in stimulated secretion (21), even though SNAP23 functions in both processes (20). What might be the adaptive value of using different exocytic machines for baseline and stimulated secretion rather than using a single machine capable of running at different rates? In view of the differing roles of baseline and stimulated mucin secretion in mucociliary clearance and airway occlusion (12), and the differing roles of Muc5ac and Muc5b in helminthic and microbial defense $(4,10)$, several plausible possibilities exist (49). These include different exocytic machines acting on small immature granules in baseline secretion to minimize the chance of airway occlusion and on large mature granules in stimulated secretion to maximize the chance of occlusion. This might occur by exchanging VAMPs during granule maturation, by analogy with Rab conversion during progression from early to late endosomes (50). Another possibility is that Muc5ac and Muc5b are packaged separately during exit from the trans-Golgi network (51), with different exocytic machines acting on secretory granules containing either secreted mucin. This last possibility is supported by the apparent segregation of MUC5AC and MUC5B extracellularly and intracellularly in human asthmatic airways (52). Further studies colocalizing vesicular components of the exocytic machinery, such as VAMPs and synaptotagmins, with different secreted mucins will be required to resolve these questions. The fact that secretory granules visualized by electron microscopy in Munc18a/b-double deletant mice are smaller and denser than granules in WT mice (Supplemental Figure 5) suggests that these SM proteins also mediate homotypic fusion between granules during post-Golgi maturation and that mucins decondense to some degree during granule maturation. Analysis of single-deletant mice suggests that Munc18a might play a greater role than Munc18b during granule biogenesis (Supplemental Figure 5), but precise delineation of their respective roles will require further studies directed specifically at this question.

Importantly, the existence of 2 exocytic machines in airway secretory cells affords the possibility to molecularly target the pathologic consequences of stimulated mucin secretion without compromising the critical protective clearance function of baseline mucin secretion. Although the fundamental pathophysiologic processes in allergic asthma and cystic fibrosis are mucin hyperproduction and impaired anion transport, respectively, stimulated mucin secretion contributes to airway occlusion in both diseases, as indicated by our prior (12) and current studies in mouse models of allergic asthma (Figure 5) and cystic fibrosis (Figure 6).

To fully appreciate the contribution of stimulated secretion to airway mucus occlusion, it is important to recognize several features of mucin biology (49). First, the production of mucins, particularly of Muc5ac, can be greatly increased by inflammatory signaling, resulting in the filling of airway epithelial secretory cells with large amounts of mucin contained within secretory granules (Figure 3 ). If secretion is not stimulated, the stored mucins are slowly released and cause only minimal mucus occlusion (12). Second, stimulated secretion can result in the explosive exocytic release of mucin granule contents within seconds (18), and the massive extracellular swelling of the released mucins by absorbing several hundred-fold their mass of water occurs in less than a second $(3,53)$. Thus, the facts that the area of the airway cross-section occupied by intracellular mucin is only a small fraction of that occupied by fully hydrated extracellular mucus, and that the extracellular expansion of mucin volume occurs within such a short time frame, are key determinants of pathophysiology.

In large proximal airways, rapid release and expansion of stored mucins from surface epithelial cells is unlikely to completely occlude the airway lumen and may have adaptive value in promoting the trapping of particles and pathogens for clearance, as described for submucosal glands (54-56). However, in small distal airways, rapid massive mucin release can overwhelm clearance mechanisms, resulting in airway lumenal occlusion (ref. 12 and Figures 5 And 6). The adaptive value of small airway occlusion in the stimulated secretion of metaplastic epithelial cells may be to trap helminths migrating through lungs (11), a hypothesis being tested by us and others. In allergic asthma, IL-13 plays a central role in increased mucin production, as it does in helminth infection, and rapid secretion can be induced by acute inflammatory mediators, such as acetylcholine and histamine $(11,12)$. In cystic fibrosis, increased mucin production is more modest (32), and the role of secretagogues in small distal airways is less well studied. However, the stimulation of 
secretion from submucosal glands is critical to mucus dysfunction in a pig model of cystic fibrosis (32), and the stimulation of mucin secretion from surface epithelial cells by acetylcholine from neurons or ATP from leukocytes or epithelial cells could contribute to mucus occlusion in small airways.

Mucus occlusion was significantly reduced by deletion of Munc18b in airway epithelial cells in both of the mouse models of airway disease we tested. In the allergic asthma model, reduction of mucus occlusion was shown to result in improvement of lung mechanics (Figure 5, C and D). In the cystic fibrosis model, while the emphysema that occurs secondary to mucus occlusion was also mitigated (Figure 6, A and C), neutrophilic and eosinophilic inflammation were not reduced (Figure 6, D), similar to what occurred upon treatment with aerosolized hypertonic saline solution in that model (57). Deletion of Munc18b in airway epithelium did not result in any abnormalities of lung structure, particle clearance, inflammation, or bacterial infection (Supplemental Figure 6). Therefore, therapeutically targeting the stimulated exocytic machine with small molecules or RNA-silencing technologies in humans might be free of intrinsic adverse consequences.

\section{Methods}

Mice. C57BL/6J (catalog 000664), C57BL/6-Tg(Zp3-cre)93Knw/J (catalog 003651), Gt(ROSA)26Sor m4(ACTB-tdTomato,-EGFP)Luo/J (catalog 007576), and B6N.129S6(Cg)-Scgb1a1 $1^{\text {tm1(cre/ERT)Blh} / J ~(c a t a l o g ~ 016225) ~}$ mice were purchased from The Jackson Laboratory. We obtained Munc18a-conditional deletant mice from Matthijs Verhage (27), Munc18c-conditional deletant mice from Roberto Adachi (26) and CCSPicre $\left(\mathrm{Scgb1a} 1^{\mathrm{tm} 1(\mathrm{icr}) \mathrm{Fj}}\right)$ mice from Francisco DeMayo (National Institute of Environmental Health Sciences, Durham, North Carolina, USA) (28). $\beta$-ENaC C57BL/6-Tg mice (35) were crossed to Munc18b-conditional deletant mice at the University of North Carolina at Chapel Hill.

In the Munc18a-conditional deletant phenotype, exon 2 of the gene is flanked by 2 loxP sites and Cre recombination induces a frameshift resulting in a nonsense codon and absence of protein (27). In the Munc18c-conditional deletant phenotype, exon 1 is flanked by 2 loxP sites and Cre recombination removes the start codon (26). To generate Munc18b-conditional deletant mice, we built a targeting vector to insert 2 loxP sites to flank exon 1 (Supplemental Figure 1) by homologous recombination. Upstream of exon 1, the zeocin and puromycin resistance genes flanked by 2 loxP sites were inserted, and downstream of exon 1, the phosphoglucokinase promoter-neomycin resistance gene flanked by 2 FRT sites was inserted. The herpes simplex virus thymidine kinase gene was introduced outside the homology arms for a negative selection marker. This vector was electroporated into 129S6:B6 embryonic stem cells (a gift from Dr. Andras Nagy, Lunenfeld-Tanenbaum Research Institute, Mount Sinai Hospital, Toronto, Canada); after 24 hours cells were selected using 1-(2-deoxy-2-fluoro-1-D-arabinofuranosyl)-5-iodouracil (Moravek Biochemicals), puromycin, and G418. Of 84 surviving clones, 1 correctly targeted clone was chosen for subsequent manipulation. The puromycin resistance gene was removed in vitro by electroporation of a circular CMV-Cre plasmid into the positive clone. Five dilution plates were prepared in order to select individual clones. Cells were plated at low density with $\times 2$ dilution from one plate to the next to reduce cell density from $2.5 \times 10^{5}$ to $2.0 \times 10^{4} \mathrm{cells} / \mathrm{ml}$. Individual clones were transferred to 96 -well plates. Duplicate plates were prepared, and 1 plate was selected on puromycin. Of the 19 subclones that did not survive puromycin selection, 1 subclone was shown to have correct targeting to remove the puromycin resistance gene by PCR. This subclone was used for injection into B6(Cg)-Tyr $\mathrm{C}^{-2 \mathrm{~J}} / \mathrm{J}$ blastocysts. The 8 chimeric males generated were crossed to B6(Cg)-Tyrc-Gt(ROSA)26Sor ${ }^{\text {tm1(FLP1)Dym/RainJ }}$ (The Jackson Laboratory, catalog 009086) to remove PGK-Neo and establish our floxed line. We then crossed the Munc18b-floxed mouse with C57BL/6-Tg(Zp3-cre)93Knw/J (catalog 003651) to generate full-animal knockouts (Supplemental Table 1) or with CCSPicre mice for specific deletion in the airway epithelium. All our lines were crossed with C57BL/6J mice for 10 generations.

Genotypes of Munc18b mutant mice were determined by PCR of genomic DNA with primers 7 (AAGGCGGTGGTAGGGAAAGT) and 64 (CAGTTGGTCAAATTCAAGTGCTC) (Figure 1A) to differentiate between the conditional (F; 1,075 bp) and WT (+; $931 \mathrm{bp}$ ) alleles. Munc18a- and Munc18c-mutant mice were genotyped as previously described (26). The presence of Zp3-Cre was determined by PCR with primers ZpCre 5' (GCGGTCTGGCAGTAAAAACTTC), ZpCre 3' (GTGAAACAGCATTGCTGTCACTT), IntControl 5' (CTAGGCCACAGAATTGAATTGAAAGATCT), and IntControl 3' (GTAGGTGGAAATTCTAGCATCATCC), which give a 324-bp internal control band and a 100-bp band from the transgene. The presence of conditional $\mathrm{CCSP}^{\mathrm{iCre}}$ was determined by PCR with primers CC10-iCreR 
(GAGATGTCCTTCACTCTGATTC); CC10-iCreF (TCTGATGAAGTCAGGAAGAACC), FJD13 (TGCCAGAGATTGTTCTAGAAAACAA), and FJD14 (GGCACAATGATGTTAATGACGTAAA), which give a $1-\mathrm{kbp}$ internal control band and a $0.5-\mathrm{kbp}$ band from the transgene. Genotyping for the $\beta$-ENaC-Tg mice was performed as previously described (35).

For CreER induction, 5 doses of $6 \mathrm{mg}$ of tamoxifen (T5648, MilliporeSigma) dissolved in corn oil (C8267, MilliporeSigma) were injected into adult mice every other day i.p. Mice lungs were harvested 2 days after the last dose. Mice of both sexes were used in all experiments, ranging from 6 to 32 weeks of age.

Immunohistochemistry. Lungs were inflated at $20 \mathrm{~cm}$ of water pressure and fixed with $10 \%$ neutral buffered formalin (NBF) for 24 hours at $4^{\circ} \mathrm{C}$ and then embedded in paraffin. Lung sections were cut into $5-\mu \mathrm{m}$ transverse sections, deparaffinized, exposed for 10 minutes to $3 \% \mathrm{H}_{2} \mathrm{O}_{2}$ in $90 \%$ methanol, and then heated for 10 minutes in $10 \mathrm{mM}$ sodium citrate, $\mathrm{pH}$ 6.0, for antigen retrieval. Tissue sections were blocked with $5 \%$ donkey serum (017-000-121, Jackson ImmunoResearch) for 1 hour at room temperature and then incubated with goat anti-CCSP (a gift from Barry Stripp, Cedars-Sinai, Los Angeles, California, USA; 1:2,000) diluted in blocking solution at $4^{\circ} \mathrm{C}$ overnight. Secondary antibody HRP-labeled donkey anti-goat (705-035003, Jackson ImmunoResearch; 1:500) was incubated for 1 hour at room temperature. Tissue sections were then washed with PBS, dehydrated, and mounted with VectaMount (Vector Laboratories).

In situ hybridization and immunofluorescence. In situ RNA detection was performed using the RNAscope detection kit (Advanced Cell Diagnostics) according to the manufacturer's instructions. Briefly, lungs were inflated at $20 \mathrm{~cm}$ of water pressure and fixed with $10 \%$ NBF for 30 hours at room temperature and then embedded in paraffin. Tissue blocks were cut into $5-\mu \mathrm{m}$ sections, deparaffinized, and pretreated with heat and protease before hybridization with the target oligonucleotide probes: murine Munc18a (Probe-MmStxbp1, 521961), Munc18b (Probe-Mm-Stxbp2, 536201), and Munc18c (Probe-Mm-Stxbp3, 536191). The positive control probe was peptidylprolyl isomerase B (catalog 313911) and the negative control probe was 4-hydroxy-tetrahydrodipicolinate reductase (from Bacillus subtilis, catalog 310043, Advanced Cell Diagnostics). Preamplifier, amplifier, and fluorescent-labeled oligonucleotides were then hybridized sequentially. RNAscope signal was imaged at the axial bronchus, between lateral branches 1 and 2 (30). Images were acquired using a confocal microscope (A1plus, Nikon) with a $\times 40$ NA 1.3 objective lens. For analysis, the number of dots per secretory, ciliated cell, or subepithelial section were counted using ImageJ $(\mathrm{NIH})(58,59)$. Because dot intensity represents transcript amount, brighter or bigger dots were counted twice. More than $70 \%$ of the dots were scored as singlets, so scoring all the doublets as singlets could result in only a $4 \%$ error.

Immunofluorescence microscopy was performed using antibodies against goat CCSP (1:2000) and mouse acetylated tubulin (T6793 MilliporeSigma, 1:2000). Secondary antibodies were species-specific donkey anti-IgG coupled with Alexa Fluor 488 (A21202) and Alexa Fluor 647 (A21447) (1:1,000, Invitrogen).

Airway epithelial mucin content by PAFS staining and image analysis. Mucous metaplasia was induced in the airways of mice by sensitizing them to OVA (20 $\mu$ g OVA grade V, $2.25 \mathrm{mg}$ alum in $0.9 \%$ saline, pH 7.4, MilliporeSigma) administered by i.p. injection once weekly for 3 weeks. Sensitized mice were exposed for 20 minutes to an aerosol challenge of $2.5 \%$ (wt/vol) OVA in $0.9 \%$ saline supplemented with $0.02 \%$ (vol/vol) antifoam A silicon polymer (MilliporeSigma) daily for 5 days via an AeroTech II nebulizer (Biodex) driven by $10 \mathrm{~L} / \mathrm{min}$ of a mixture of $95 \%$ room air supplemented with $5 \% \mathrm{CO}_{2}$ to induce deep ventilation. Three days after the last OVA aerosol exposure, half of the mice in each group were exposed for 10 minutes to an aerosol of $100 \mathrm{mM}$ ATP (MilliporeSigma) in $0.9 \% \mathrm{NaCl}$ to induce mucin secretion, then sacrificed after 20 minutes. Lungs were harvested, inflated, and then fixed in $10 \% \mathrm{NBF}$, embedded in paraffin, and sectioned into a single transverse 5 - $\mu \mathrm{m}$ cut of the axial airway of the left lung, between lateral branches 1 and 2 (30). Sections were deparaffinized, rehydrated, and then stained with PAFS. Images were acquired using an upright microscope (Olympus BX 60) with a $\times 40$ NA 0.75 objective lens, and intracellular mucin was measured around all the circumferential section of the axial bronchus using ImagePro (Media Cybernetics). Data are presented as the epithelial mucin volume density, signifying the measured volume of mucin overlying a unit area of epithelial basal lamina, derived as described previously (60). Images were analyzed by investigators blinded to mouse genotype and treatment.

Agarose gel Western blot analysis for mucin. Lungs were perfused by intracardiac injection of $2 \mathrm{ml}$ PBS until blanched, then homogenized in $1 \mathrm{ml}$ of 6-M guanidinium buffer containing protease inhibitors (MilliporeSigma) and incubated for 2 days at $4^{\circ} \mathrm{C}$. Lysates were centrifuged at 19,000 $\mathrm{g}$ and the supernatants were then dialyzed overnight at $4^{\circ} \mathrm{C}$ against PBS in Slide-A-Lyzer $2 \mathrm{~K}$ MWCO 3-ml cassettes (Thermo Fisher Scientific). Protein concentrations were determined using a bicinchoninic acid protein assay kit (Thermo Fisher Scientific), and then the samples were incubated with 20-Kunitz DNase (LS002139, Worthington) 
for 15 minutes at $37^{\circ} \mathrm{C}$ and reduced with $10 \mathrm{mM}$ dithiothreitol (MilliporeSigma) for 10 minutes at $95^{\circ} \mathrm{C}$ in loading buffer ( $5 \%$ glycerol, $0.1 \%$ SDS, $0.0025 \%$ bromophenol blue, $0.6 \mathrm{M}$ urea, $10 \mathrm{mM}$ Tris- $\mathrm{HCl}$, and 0.5 mM EDTA, $\mathrm{pH}$ 8). Samples were electrophoresed through a $0.8 \%$ agarose $/ 0.1 \%$ SDS hydrogel, and the gel was then soaked in $10 \mathrm{mM}$ dithiothreitol for 20 minutes. Then the samples were transferred by vacuum onto a PVDF membrane. Membranes were washed in PBS and blocked with 5\% nonfat milk in PBS/0.05\% Tween before probing with lectin UEA-1 conjugated to $\operatorname{HRP}(1: 1,000$, L8146, MilliporeSigma) in blocking solution to detect fucosylated Muc5ac (30) or with monoclonal antibody MDA-3E1 raised by us against peptide TTCQPQCQWTKWIDVDYPSS in blocking solution $(1: 1,500)$ to detect Muc5b (20). Secondary antibody against Muc5b was goat anti-mouse conjugated to HRP (1:5,000, Thermo Fisher Scientific), and the chemiluminescence signal was detected with Pierce ECL Western Blotting Substrate (Thermo Fisher Scientific). Relative protein amounts were determined for each sample using ImageJ (NIH) (59) against a standard curve run for each gel (Supplemental Figure 4).

Electron microscopy and stereology. Slight mucous metaplasia was induced in mice with 1 dose of $0.2 \mu \mathrm{g}$ of IL-13 instilled intrapharyngeally on day 1 of the experiment to achieve optimal visualization of mucin granules. Mice were then anesthetized and sacrificed on day 5. Lungs were excised and fixed in $2.5 \%$ glutaraldehyde in 0.1-M sodium cacodylate buffer ( $\mathrm{pH}$ 7.2) containing $20 \mathrm{mM}$ calcium chloride for 2 hours, followed by a 1 -hour subsequent fixation in buffered $1 \%$ osmium tetroxide. The fixed left lung was then sectioned into a single transverse cut of the axial airway between lateral branches 1 and 2 and embedded in EMbed 812 epoxy resin (14120, Electron Microscopy Sciences). Sections of 100-nm thickness were stained with uranyl acetate and lead citrate and were viewed at $\times 8,200$ magnification in a Tecnai 12 transmission electron microscope. Secretory cells were randomly selected and imaged, and at least 19 images were used per mouse. SvG was obtained by stereology with dot-and-line grids (line pairs, 64 tiles) randomly placed on the cell profiles (61). Granule number was calculated by counting all mucin granules in the cytoplasmic area of secretory cells using ImageJ (NIH) (59). To measure the relative electron lucency of mucin granules, a cycloid stereologic grid with circles of 30 pixels in diameter was randomly superimposed on the images. At least 5 circles that fell on nuclear heterochromatin and electron-lucent extracellular space were selected, and their grayscale value (from 0 to 255) was recorded. These values were used to set a linear scale for each image. Then at least 30 random circles that fell on granules were recorded using that scale. These data were recorded and the appropriate bin range and histogram distribution were calculated as described previously (62).

Mucin transcript quantitative reverse transcription PCR analysis. Total RNA was extracted from whole lung using an RNeasy mini kit (Qiagen) or from purified cells using Trizol reagents (Invitrogen) and an RNeasy micro kit (Qiagen), then reverse-transcribed using iSCRIPT cDNA synthesis kit (Bio-Rad). qPCR was carried out for each cDNA sample in triplicate with qPCR master mix (Quanta BioSciences) and 6-carboxyfluorescein-labeled probes for Muc5b (Mm00466391_m1), Muc5ac (Mm01276725_g1), Munc18a (Mm00436837_m1), Munc18b (Mm00441589_m1), Munc18c (Mm00441605_m1), and $\beta$-actin (Mm02619580_g1, all from Thermo Fisher Scientific) on a ViiA 7 Real-Time PCR System (Applied Biosystems). Results were expressed as $\Delta \mathrm{Ct}$ (normalized for $\beta$-actin) (63).

FACS of airway epithelial cells. Lungs from CCSP $\mathrm{P}^{\mathrm{iCr} /+} \mathrm{ROSA}^{\mathrm{mTmG} / \mathrm{mTmG}}$ alone or crossed with Mun-

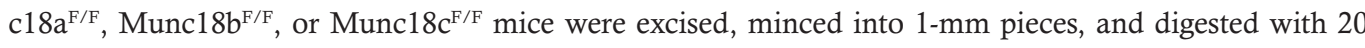
$\mathrm{mg} / \mathrm{ml}$ collagenase type I (Worthington) and $5 \mathrm{mg} / \mathrm{ml}$ DNase I (Worthington) for 1 hour at $37^{\circ} \mathrm{C}$ with frequent agitation. An equal volume of $0.25 \%$ Trypsin-EDTA (Gibco) was added and incubated for another 10 minutes at $37^{\circ} \mathrm{C}$. After digestion, FBS (Gibco) was added to a final concentration of $15 \%$ (vol/vol), and the samples were further triturated by pipetting 30 times. Dissociated cells were washed with PBS with $1 \%$ FBS and sorted on a BD Biosciences Influx sorter.

Lung lavage. This was performed by instilling and collecting $1 \mathrm{ml}$ of PBS through a tracheostomy (20-gauge cannula). Total leukocyte count was determined using a hemocytometer and differential count by cytocentrifugation of $200 \mu \mathrm{l}$ of lavage fluid at $300 \mathrm{~g}$ for 5 minutes. Cytospins were stained by Wright-Giemsa for microscopic cell identification and counting.

Mucociliary clearance. Mucociliary clearance was measured as the elimination of fluorescent microspheres from the lungs over time. Mice were anesthetized with urethane ( $2 \mathrm{mg} / \mathrm{g}, \mathrm{i} . \mathrm{p}$.) and tracheostomized with a blunt beveled 18-gauge Luer-Stub adapter (Becton, Dickinson). Using a microsprayer (Penn-Century), $25 \mu$ of PBS/ $0.1 \%$ Tween containing $7.43 \times 10^{4}$ of $4.19-\mu \mathrm{m}$ fluorescent microspheres (Bangs Laboratories Inc.) were loaded at the lung carina through the tracheostomy. Lungs were harvested immediately (time 0 ), or mice were mechanically ventilated with a flexiVent (Scireq) to guarantee uniform ventilation. 
Lungs were harvested after 30 minutes and homogenized with $1.5 \mathrm{~g}$ of $1.3-\mathrm{mm}$ chrome steel beads (BioSpec) and $1 \mathrm{ml}$ of PBS/0.1\% Tween using a Mini-Bead Beater (BioSpec) at 4,800 rpm for 3 minutes. Fluorescent microspheres were then manually counted using a hemocytometer.

Total bacterial $16 S$ rDNA qPCR. Lungs were harvested and bacterial genomic DNA was extracted and analyzed at Baylor College of Medicine by methods developed for the NIH Human Microbiome Project $(64,65)$. Briefly, bacterial genomic DNA was extracted using a PowerSoil DNA Isolation Kit (MO BIO Laboratories) following the manufacturer's instructions. Extracted DNA concentrations were measured by Qubit (Life Technologies) for subsequent normalization of qPCR results. qPCR sample analysis was performed in a 7500 Fast RealTime PCR System (Applied Biosystems). The qPCR primers (1369F-1492R) target regions flanking V9 of the 16S rRNA gene (66). A standard curve was made using a serially diluted plasmid that contains nucleotides 1369 to 1492 of an Escherichia coli $16 \mathrm{~S}$ rRNA gene, and concentrations of the samples were calculated from $\mathrm{C}_{\mathrm{T}}$ values using the equation generated from plotting the standard curve. All samples were run in triplicate, including the standard curve, a set of nontemplate controls, and inhibitor controls (known positives plus unknown DNA).

Analysis of $16 S$ rRNA gene composition. The $16 \mathrm{~S}$ rDNA V4 region was amplified by PCR and sequenced in the MiSeq platform (Illumina) using the $2 \times 250 \mathrm{bp}$ paired-end protocol yielding pair-end reads that overlap almost completely. The primers used for amplification contain adapters for MiSeq sequencing and dual-index barcodes so that the PCR products may be pooled and sequenced directly (67), targeting at least 10,000 reads per sample. The read pairs are demultiplexed based on the unique molecular barcodes, and reads are merged using USEARCH v7.0.1001 (68), allowing 0 mismatches and a minimum overlap of 50 bases. Merged reads are trimmed at first base with Q5. In addition, a quality filter is applied to the resulting merged reads, and reads containing above 0.05 expected errors are discarded. 16S rRNA gene sequences were assigned into operational taxonomic units (OTUs) or phylotypes at a similarity cutoff value of $97 \%$ using the UPARSE algorithm. OTUs were then mapped to an optimized version of the SILVA database $(69,70)$ containing only the $16 \mathrm{~S} v 4$ region to determine taxonomies. Abundances were recovered by mapping the demultiplexed reads to the UPARSE OTUs.

Lumenal occlusion in an allergic asthma model. Airway mucus plugging was measured through modifications, as follows, of a method we have described previously (12). Lungs were fixed by immersion, to avoid displacement of lumenal mucus by inflation, in methanol-based Carnoy's solution (methacarn), to minimize changes in mucus volume, for 48 hours at $4^{\circ} \mathrm{C}$. Lungs were then excised, and the right caudal lobe (Figure 6B and Supplemental Figure 7B), or every lobe (Supplemental Figure 7A), was embedded in paraffin. For Supplemental Figure 7A and Supplemental Figure 7B, a 5- $\mu \mathrm{m}$ section was obtained every $500 \mu \mathrm{m}$ starting from the most caudal point of the lobe. For Figure $6 \mathrm{~B}$, a 5 - $\mu \mathrm{m}$ section was obtained every $1,000 \mu \mathrm{m}$ of the right caudal lobe, yielding 4 sections per lung. Slides were then deparaffinized, rehydrated, and stained with PAFS. Images were acquired using an upright microscope (Olympus BX 60) with a $\times 20$ NA 0.5 lens objective. For quantification, the cross-sectional area of the lumenal mucus was traced (Figure 6B), and the airway cross-sectional area was traced to calculate the occlusion fraction (Supplemental Figure 7, A and B), using ImageJ (NIH) (59).

Lung mechanics. Respiratory resistance was analyzed using a flexiVent system (Scireq). Mice were anesthetized with urethane ( $3 \mathrm{mg} / \mathrm{g}$ by i.p. injection, a dose sufficient for 2 hours of sedation even though experiments last less than 30 minutes) and paralyzed with succinylcholine chloride ( $5 \mathrm{mg}$ by i.p. injection followed by continuous i.p. infusion at $20 \mu \mathrm{g} / \mathrm{g} \cdot \mathrm{min})$. Mice were tracheostomized with a blunt beveled 18-gauge Luer-Stub adapter and ventilated at $150 \mathrm{breaths} / \mathrm{min}, 10 \mu \mathrm{l} / \mathrm{g}$, against $2-3 \mathrm{~cm} \mathrm{H}_{2} \mathrm{O}$ positive end-expiratory pressure. Respiratory resistance was assessed at baseline and in response to 4 incremental doses of Mch (1, 3,10 , and $30 \mathrm{mg} / \mathrm{ml}$ ) administered by an in-line ultrasonic nebulizer (4-6 $\mu \mathrm{m}$, Aerogen). Total respiratory resistance was calculated by averaging 8 values measured after each dose of Mch for each mouse.

Lumenal occlusion and emphysema in the $\beta$-ENaC-Tg model of cystic fibrosis. Lungs were fixed with $10 \%$ $\mathrm{NBF}$, and the left lobes were cut in transverse sections starting at the level of the hilum and then every 2 $\mathrm{mm}$ from rostral to caudal, yielding 2 to 4 slices. All slices were embedded in paraffin, and a 5 - $\mu \mathrm{m}$ section was obtained from each slice. Sections were then stained with AB-PAS (71). Whole lung section images were obtained in an Olympus BX61VS scanner with a BX81 stage and UPlanSApo $\times 20$ NA 0.75 objective lens; scanning conditions were kept constant among specimens. Lumenal occlusion was quantified as in Figure 5B from the first 2 sections of each mouse. Emphysema was measured using $\mathrm{D}_{2}$, computed from measurement of airspace area and weighted for variance and for skewness toward large spaces (72), from 10 high-magnification noncontinuous images $(1134 \times 1134$ pixels $)$, equidistant from the center of the section and excluding airways, selected from the sections used in Figure 6B. 
Statistics. All statistical analyses were performed using GraphPad Prism 7.0, with $P<0.05$ considered statistically significant. Exact $P$ values and $n$ values for each sample are included in each figure legend. Statistical analysis was performed using 1-way ANOVA followed by Tukey's post hoc test for multiple pairwise comparisons and Student's 2-tailed $t$ test or Mann-Whitney $U$ test after determining normality of the data using D'Agostino-Pearson omnibus K2 test. A Kolmogorov-Smirnov test was used for Supplemental Figure 5D. Values that did not reach significance were not noted. After determining that the expression and secretory function of Munc18 proteins in floxed mice was equivalent to WT, the primary endpoint for studies of secretion, clearance, and obstruction was of differences between airway-deletant mice and their floxed littermates. For box plots, lines represent medians; boxes represent 25 th -75 th percentiles; and whiskers represent 5 th-95th percentiles.

Study approval. All mice were kept in specific pathogen-free facilities and handled in accordance with the protocol approved by the Institutional Animal Care and Use Committees of The University of Texas MD Anderson Cancer Center (00001214-RN01), The Texas A\&M University Health Science Center Institute of Bioscience and Technology (IAUCUC 2017-0121-IBT) and The University of North Carolina (IAUCUC 15-338).

\section{Author contributions}

MJT and BFD conceived the study. AMJ performed most experiments and analyses. LP, WVV, ASHD, ZA, UN, JF, JB, and JPT conducted the remaining experiments. MJT and BLS generated the conditional Munc18b mouse. RA generated the conditional Munc18c mouse and helped with statistical and stereologic analyses. CME generated the Muc5ac-knockout mouse and assisted with the analysis of mucus occlusion. SMK and FC performed the mouse crossing/breeding, experimental procedures, and data analysis in the $\beta$-ENaC-Tg studies. ARB performed the electron microscopy. AMJ and BFD wrote the manuscript. All authors contributed to discussion of the study.

\section{Acknowledgments}

We thank Matthijs Verhage (University of Amsterdam) for reagents and helpful discussion, Mehmet Kesimer (UNC-CH) for advice with mucin immunoblots, David E. Ost for assistance with statistical methods, James P. Carson for facilitating use of the program for emphysema analysis, and the University of Texas MD Anderson Genetically Engineered Mouse Facility (GEMF). For the $\beta$-ENaC-Tg studies, we thank the Cystic Fibrosis Center Molecular Biology Core (W. O’Neal, UNC-CH) for the genotyping, C. van Heusden (UNC-CH) for the bronchoalveolar lavage preparations, Kim Burns (UNC-CH) for the lung histology processing, and Hong Dang for the statistical power analysis. AMJ thanks the members of her doctoral thesis committee, Magnus Hook, David Reiner, James McNew, and Margie Moczygemba, for helpful advice. This work was supported by NIH grants R01HL129795, R41HL136057, R01AI093533, National Institute of Diabetes and Digestive and Kidney Diseases DK065988, R01HL080396, R01HL130938, and R21ES023384; by Cystic Fibrosis Foundation grants DICKEY15P0, DICKEY18G0, KREDA13G0, KREDA16XX0, and BOUCHER15R0; and by Department of Defense grant PR160247. The GEMF at MD Anderson is supported by the Cancer Center Support Grant NCI-CA016672 (GEMF). BLS was supported by the MD Anderson Odyssey Fellowship program.

Address correspondence to: Ana M. Jaramillo or Burton F. Dickey, 1515 Holcombe Blvd, Houston, Texas 77030, USA. Phone: 713.563.4253; Email: anamariajh@gmail.com (A.M. Jaramillo); bdickey@mdanderson.org (B.F. Dickey).

BLS's present address is: Pulmotect Inc., Houston, Texas, USA.

1. Fahy JV, Dickey BF. Airway mucus function and dysfunction. N Engl J Med. 2010;363(23):2233-2247.

2. Thornton DJ, Rousseau K, McGuckin MA. Structure and function of the polymeric mucins in airways mucus. Annu Rev Physiol. 2008;70:459-486.

3. Verdugo P. Supramolecular dynamics of mucus. Cold Spring Harb Perspect Med. 2012;2(11):a009597.

4. Roy MG, et al. Muc5b is required for airway defence. Nature. 2014;505(7483):412-416.

5. Grubb BR, Livraghi-Butrico A, Rogers TD, Yin W, Button B, Ostrowski LE. Reduced mucociliary clearance in old mice is associated with a decrease in Muc5b mucin. Am J Physiol Lung Cell Mol Physiol. 2016;310(9):L860-L867.

6. Seibold MA, et al. A common MUC5B promoter polymorphism and pulmonary fibrosis. N Engl J Med. 2011;364(16):1503-1512

7. Evans CM, et al. Idiopathic pulmonary fibrosis: a genetic disease that involves mucociliary dysfunction of the peripheral air- 
ways. Physiol Rev. 2016;96(4):1567-1591.

8. Young HW, et al. Central role of Muc5ac expression in mucous metaplasia and its regulation by conserved $5^{\prime}$ elements. Am $J$ Respir Cell Mol Biol. 2007;37(3):273-290.

9. Zhen G, et al. IL-13 and epidermal growth factor receptor have critical but distinct roles in epithelial cell mucin production. Am J Respir Cell Mol Biol. 2007;36(2):244-253.

10. Hasnain SZ, et al. Muc5ac: a critical component mediating the rejection of enteric nematodes. J Exp Med. 2011;208(5):893-900

11. Dickey BF. Exoskeletons and exhalation. NEngl J Med. 2007;357(20):2082-2084.

12. Evans CM, et al. The polymeric mucin Muc5ac is required for allergic airway hyperreactivity. Nat Commun. 2015;6:6281

13. Davis CW, Dickey BF. Regulated airway goblet cell mucin secretion. Annu Rev Physiol. 2008;70:487-512.

14. Zhu Y, et al. Baseline Goblet cell mucin secretion in the airways exceeds stimulated secretion over extended time periods, and is sensitive to shear stress and intracellular mucin stores. PLoS One. 2015;10(5):e0127267.

15. Zhu Y, et al. Munc13-2-/- baseline secretion defect reveals source of oligomeric mucins in mouse airways. J Physiol (Lond). 2008;586(7):1977-1992.

16. Button B, Okada SF, Frederick CB, Thelin WR, Boucher RC. Mechanosensitive ATP release maintains proper mucus hydration of airways. Sci Signal. 2013;6(279):ra46.

17. Young HW, Sun CX, Evans CM, Dickey BF, Blackburn MR. A3 adenosine receptor signaling contributes to airway mucin secretion after allergen challenge. Am J Respir Cell Mol Biol. 2006;35(5):549-558.

18. Davis CW, Dowell ML, Lethem M, Van Scott M. Goblet cell degranulation in isolated canine tracheal epithelium: response to exogenous ATP, ADP, and adenosine. Am J Physiol. 1992;262(5 Pt 1):C1313-C1323.

19. Tuvim MJ, et al. Synaptotagmin 2 couples mucin granule exocytosis to $\mathrm{Ca}^{2+}$ signaling from endoplasmic reticulum. $J$ Biol Chem 2009;284(15):9781-9787.

20. Ren B, et al. SNAP23 is selectively expressed in airway secretory cells and mediates baseline and stimulated mucin secretion. Biosci Rep. 2015;35(3):e00220.

21. Jones LC, et al. VAMP8 is a vesicle SNARE that regulates mucin secretion in airway goblet cells. J Physiol (Lond). 2012;590(3):545-562.

22. Rizo J, Xu J. The synaptic vesicle release machinery. Annu Rev Biophys. 2015;44:339-367.

23. Kim K, et al. Munc18b is an essential gene in mice whose expression is limiting for secretion by airway epithelial and mast cells Biochem J. 2012;446(3):383-394.

24. Verhage M, et al. Synaptic assembly of the brain in the absence of neurotransmitter secretion. Science. 2000;287(5454):864-869.

25. Oh E, Spurlin BA, Pessin JE, Thurmond DC. Munc18c heterozygous knockout mice display increased susceptibility for severe glucose intolerance. Diabetes. 2005;54(3):638-647.

26. Gutierrez BA, et al. Munc18-2, but not Munc18-1 or Munc18-3, controls compound and single-vesicle-regulated exocytosis in mast cells. J Biol Chem. 2018;293(19):7148-7159.

27. Heeroma JH, et al. Trophic support delays but does not prevent cell-intrinsic degeneration of neurons deficient for munc18-1. Eur J Neurosci. 2004;20(3):623-634.

28. Liu J, et al. ErbB2 pathway activation upon Smad4 loss promotes lung tumor growth metastasis. Cell Rep. 2015;10(9):1599-1613.

29. Rawlins EL, et al. The role of Scgbla1+ Clara cells in the long-term maintenance and repair of lung airway, but not alveolar, epithelium. Cell Stem Cell. 2009;4(6):525-534.

30. Piccotti L, Dickey BF, Evans CM. Assessment of intracellular mucin content in vivo. Methods Mol Biol. 2012;842:279-295

31. Nguyen LP, et al. Chronic exposure to beta-blockers attenuates inflammation and mucin content in a murine asthma model. $A m$ J Respir Cell Mol Biol. 2008;38(3):256-262.

32. Stoltz DA, Meyerholz DK, Welsh MJ. Origins of cystic fibrosis lung disease. N Engl J Med. 2015;372(4):351-362.

33. Henderson AG, et al. Cystic fibrosis airway secretions exhibit mucin hyperconcentration and increased osmotic pressure. JClin Invest. 2014;124(7):3047-3060

34. Kreda SM, Davis CW, Rose MC. CFTR, mucins, and mucus obstruction in cystic fibrosis. Cold Spring Harb Perspect Med. 2012;2(9):a009589.

35. Mall M, Grubb BR, Harkema JR, O’Neal WK, Boucher RC. Increased airway epithelial Na ${ }^{+}$absorption produces cystic fibrosis-like lung disease in mice. Nat Med. 2004;10(5):487-493.

36. Riento K, Kauppi M, Keranen S, Olkkonen VM. Munc18-2, a functional partner of syntaxin 3, controls apical membrane trafficking in epithelial cells. J Biol Chem. 2000;275(18):13476-13483.

37. Vogel GF, Klee KM, Janecke AR, Müller T, Hess MW, Huber LA. Cargo-selective apical exocytosis in epithelial cells is conducted by Myo5B, Slp4a, Vamp7, and Syntaxin 3. J Cell Biol. 2015;211(3):587-604.

38. Dulubova I, Khvotchev M, Liu S, Huryeva I, Südhof TC, Rizo J. Munc18-1 binds directly to the neuronal SNARE complex. Proc Natl Acad Sci U S A. 2007;104(8):2697-2702.

39. Deák F, et al. Munc18-1 binding to the neuronal SNARE complex controls synaptic vesicle priming. J Cell Biol. 2009;184(5):751-764.

40. van Breevoort D, et al. STXBP1 promotes Weibel-Palade body exocytosis through its interaction with the Rab27A effector Slp4-a. Blood. 2014;123(20):3185-3194.

41. Rodríguez F, Zanetti MN, Mayorga LS, Tomes CN. Munc18-1 controls SNARE protein complex assembly during human sperm acrosomal exocytosis. J Biol Chem. 2012;287(52):43825-43839.

42. Naren AP, et al. Regulation of CFTR chloride channels by syntaxin and Munc18 isoforms. Nature. 1997;390(6657):302-305

43. Lam PP, et al. Munc18b is a major mediator of insulin exocytosis in rat pancreatic $\beta$-cells. Diabetes. 2013;62(7):2416-2428.

44. Oh E, Kalwat MA, Kim MJ, Verhage M, Thurmond DC. Munc18-1 regulates first-phase insulin release by promoting granule docking to multiple syntaxin isoforms. J Biol Chem. 2012;287(31):25821-25833.

45. Lopez JA, et al. Bi-allelic mutations in STXBP2 reveal a complementary role for STXBP1 in cytotoxic lymphocyte killing. Front Immunol. 2018;9:529.

46. Torres J, Funk HM, Zegers MM, ter Beest MB. The syntaxin $4 \mathrm{~N}$ terminus regulates its basolateral targeting by munc18c-dependent and -independent mechanisms. J Biol Chem. 2011;286(12):10834-10846. 
47. Dolai S, et al. Depletion of the membrane-fusion regulator Munc18c attenuates caerulein hyperstimulation-induced pancreatitis. J Biol Chem. 2018;293(7):2510-2522.

48. Thurmond DC, Kanzaki M, Khan AH, Pessin JE. Munc18c function is required for insulin-stimulated plasma membrane fusion of GLUT4 and insulin-responsive amino peptidase storage vesicles. Mol Cell Biol. 2000;20(1):379-388.

49. Jaramillo AM, Azzegagh Z, Tuvim MJ, Dickey BF. Airway Mucin Secretion. Ann Am Thorac Soc. 2018;15(Supplement_3):S164-S170.

50. Rink J, Ghigo E, Kalaidzidis Y, Zerial M. Rab conversion as a mechanism of progression from early to late endosomes. Cell. 2005;122(5):735-749.

51. Pakdel M, von Blume J. Exploring new routes for secretory protein export from the trans-Golgi network. Mol Biol Cell. 2018;29(3):235-240

52. Bonser LR, Zlock L, Finkbeiner W, Erle DJ. Epithelial tethering of MUC5AC-rich mucus impairs mucociliary transport in asthma. J Clin Invest. 2016;126(6):2367-2371.

53. Shumilov D, et al. Real-time imaging of exocytotic mucin release and swelling in Calu-3 cells using acridine orange. Methods. 2014;66(2):312-324

54. Ermund A, et al. The normal trachea is cleaned by MUC5B mucin bundles from the submucosal glands coated with the MUC5AC mucin. Biochem Biophys Res Commun. 2017;492(3):331-337.

55. Ostedgaard LS, et al. Gel-forming mucins form distinct morphologic structures in airways. Proc Natl Acad Sci U S A. 2017;114(26):6842-6847.

56. Fischer AJ, et al. Mucus strands from submucosal glands initiate mucociliary transport of large particles. JCI Insight. 2019;4(1):124863.

57. Graeber SY, Zhou-Suckow Z, Schatterny J, Hirtz S, Boucher RC, Mall MA. Hypertonic saline is effective in the prevention and treatment of mucus obstruction, but not airway inflammation, in mice with chronic obstructive lung disease. Am J Respir Cell Mol Biol. 2013;49(3):410-417.

58. Silberstein L, et al. Proximity-based differential single-cell analysis of the niche to identify stem/progenitor cell regulators. Cell Stem Cell. 2016;19(4):530-543

59. Schindelin J, et al. Fiji: an open-source platform for biological-image analysis. Nat Methods. 2012;9(7):676-682.

60. Evans CM, et al. Mucin is produced by clara cells in the proximal airways of antigen-challenged mice. Am J Respir Cell Mol Biol. 2004;31(4):382-394

61. Tschanz SA, Burri PH, Weibel ER. A simple tool for stereological assessment of digital images: the STEPanizer. J Microsc. 2011;243(1):47-59.

62. Rodarte EM, et al. Munc13 proteins control regulated exocytosis in mast cells. J Biol Chem. 2018;293(1):345-358.

63. Schmittgen TD, Livak KJ. Analyzing real-time PCR data by the comparative C(T) method. Nat Protoc. 2008;3(6):1101-1108

64. Human Microbiome Project Consortium. Structure, function and diversity of the healthy human microbiome. Nature. 2012;486(7402):207-214.

65. Human Microbiome Project Consortium. A framework for human microbiome research. Nature. 2012;486(7402):215-221

66. Suzuki MT, Taylor LT, DeLong EF. Quantitative analysis of small-subunit rRNA genes in mixed microbial populations via 5'-nuclease assays. Appl Environ Microbiol. 2000;66(11):4605-4614.

67. Caporaso JG, et al. Ultra-high-throughput microbial community analysis on the Illumina HiSeq and MiSeq platforms. ISME J. 2012;6(8):1621-1624.

68. Edgar RC. Search and clustering orders of magnitude faster than BLAST. Bioinformatics. 2010;26(19):2460-2461.

69. Edgar RC. UPARSE: highly accurate OTU sequences from microbial amplicon reads. Nat Methods. 2013;10(10):996-998.

70. Quast C, et al. The SILVA ribosomal RNA gene database project: improved data processing and web-based tools. Nucleic Acids Res. 2013;41(Database issue):D590-D596.

71. Jones LC, et al. VAMP8 is a vesicle SNARE that regulates mucin secretion in airway goblet cells. J Physiol (Lond). 2012;590(3):545-562.

72. Jacob RE, Carson JP, Gideon KM, Amidan BG, Smith CL, Lee KM. Comparison of two quantitative methods of discerning airspace enlargement in smoke-exposed mice. PLoS One. 2009;4(8):e6670. 